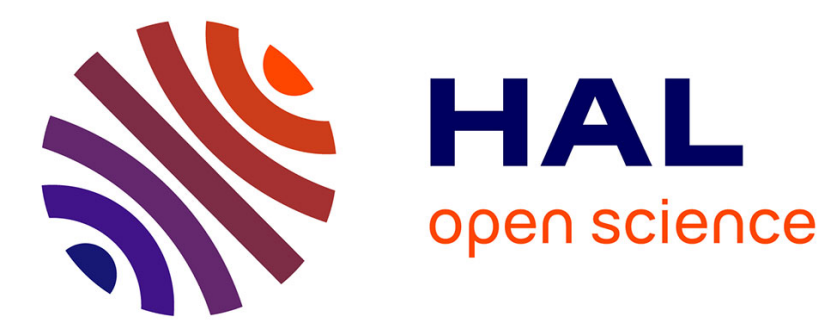

\title{
Les fouilles de la chapelle Saint-Mauront
}

\author{
Jean-Pierre Pelletier, Lucy Vallauri
}

\section{To cite this version:}

Jean-Pierre Pelletier, Lucy Vallauri. Les fouilles de la chapelle Saint-Mauront. Saint Victor de Marseille: études archéologiques et historiques, actes du colloque Saint-Victor, Marseille, 18-20 novembre 2004, Nov 2004, Marseille, France. pp.125-140. halshs-00504843

\section{HAL Id: halshs-00504843 https://shs.hal.science/halshs-00504843}

Submitted on 7 Oct 2021

HAL is a multi-disciplinary open access archive for the deposit and dissemination of scientific research documents, whether they are published or not. The documents may come from teaching and research institutions in France or abroad, or from public or private research centers.
L'archive ouverte pluridisciplinaire HAL, est destinée au dépôt et à la diffusion de documents scientifiques de niveau recherche, publiés ou non, émanant des établissements d'enseignement et de recherche français ou étrangers, des laboratoires publics ou privés. 


\section{BIBLIOTHÈQUE DE L'ANTIQUITÉ TARDIVE}

PUB LIÉE PAR L'ASSOCIATION POUR L'ANTIQUITÉ TARDIVE

\section{3}

\section{SAINT-VICTOR DE MARSEILLE ÉTUDES ARCHÉOLOGIQUES ET HISTORIQUES}

Actes du colloque Saint-Victor Marseille, 18-20 novembre 2004

Éd. Michel Fixot - Jean-Pierre Pelletier

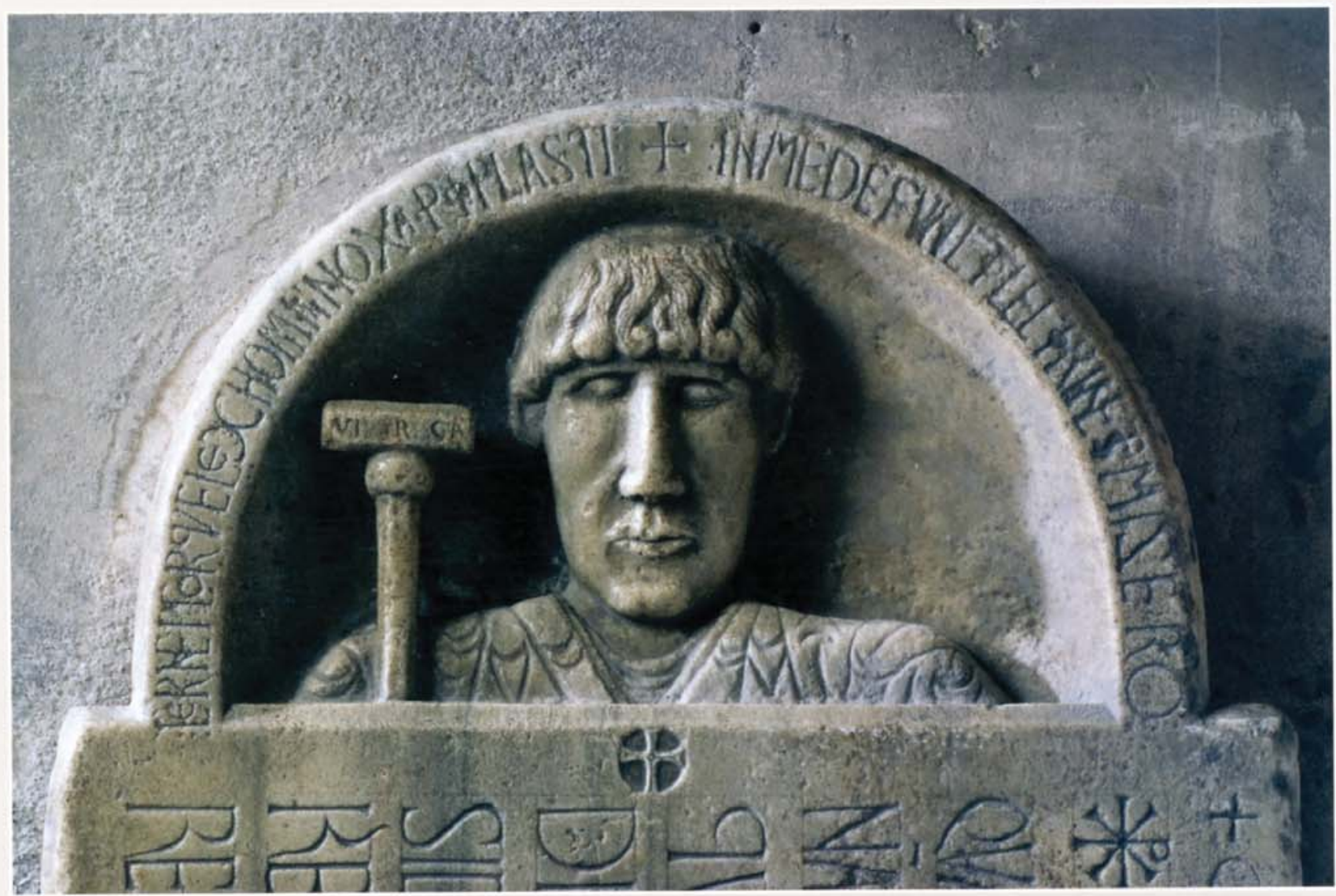




\section{LES FOUILLES DE LA CHAPELLE SAINT-MAURONT*}

Les fouilles dans cette partie du monument en 1972 ont été justifiées par la réfection complète du sol carrelé et de l'escalier descendant de la première travée de l'église, tous deux très dégradés (fig. 1). La chapelle Saint-Mauront a été établie par attaque vers l'ouest du front de taille de la carrière antique'. Cinq tombes rupestres creusées dans le plateau affleurant à la cote -300 à l'ouest des cryptes étaient encore visibles sur le pourtour, dont trois coupées sous le mur occidental de l'église (TR7, TR8, et la tombe double TR4) et deux sur le replat rocheux au nord (TR5 et TR6). Leur orientation est identique à celles observées dans la Cour ouest et la Cour sud, elles font certainement partie du même ensemble (pl. I).

Au cours de la campagne de travaux du début du XIII ${ }^{\mathrm{c}}$ siècle ${ }^{2}$, le plateau a été entaillé sur environ 8 à $9 \mathrm{~m}$ de profondeur et $40 \mathrm{~m}^{2}$ de surface par l'extension de la carrière, au-delà d'une sorte de couloir large de $2,20 \mathrm{~m}$ situé dans l'axe des piles de la première travée ( $\mathrm{pl}$. II). Dans un premier temps, le creusement effectué jusqu'au niveau du dallage des cryptes pour l'aménagement de la chapelle ( $150 \mathrm{~m}^{3}$ environ) a réservé dans le rocher, au nord et au sud, les supports des piles de la première travée de l'église. L'excavation est caractérisée par des parois présentant un fruit plus ou moins marqué dans des bancs qui présentent des intercalations de conglomérats.

\footnotetext{
* Abréviations utilisées : - Amouric et al., Vingt mille pots $=\mathrm{H}$. Amouric, F. Richez, L. Vallauri, Vingt mille pots sous les mers. Le commerce de la céramique en Provence et Languedoc du $X^{\circ}$ au $X I X^{\prime}$ siècle, Catalogue d'exposition, Musée d'Istres, Edisud, Aixen-Provence, 1999, 197 p. ;- Fixot, Pelletier, Étude archéologi$q u e=$ M. Fixot, J.-P. Pelletier, Saint-Victor de Marseille, I, Étude archéologique et monumentale ;-Marchesi et al., Marseille, les ateliers de potiers $=\mathrm{H}$. Marchesi, J. Thiriot, L. Vallauri (dir.), M. Leenhardt (coll.), Marseille, les ateliers de potiers du XIII's. et lequartier Sainte-Barbe ( $V^{e}-X V I I^{e} s$.) ( Documents d'archéologie française, 65), Paris, 1997.
}

1. Fixot, Pelletier, Étude archéologique, IX, fig. I. 2. Ibid., V, fig. 95 .

\section{A - LA CARRIÉRE}

Au-dessous d'une sorte de bordure large de 30 à $40 \mathrm{~cm}$ établie à la cote -630 , les parois de la carrière sont verticales ou en léger surplomb. Les bancs y sont plus francs, malgré la présence de fissures et de passées d'argile.

Les traces d'outils, bien conservées par un remblaiement rapide, montrent des tranches hautes de 0,25 à $0,35 \mathrm{~m}$ proches de l'horizontale, alors que le pendage naturel vers l'ouest-nord-ouest est de l'ordre de $30 \%$. L'aire d'extraction est divisée en trois chantiers secondaires, le premier au sud prolongeant le couloir d'accès, le second au centre avec des dimensions comparables, et le troisième au nord, plus étroit à cause de la retombée des doubleaux.

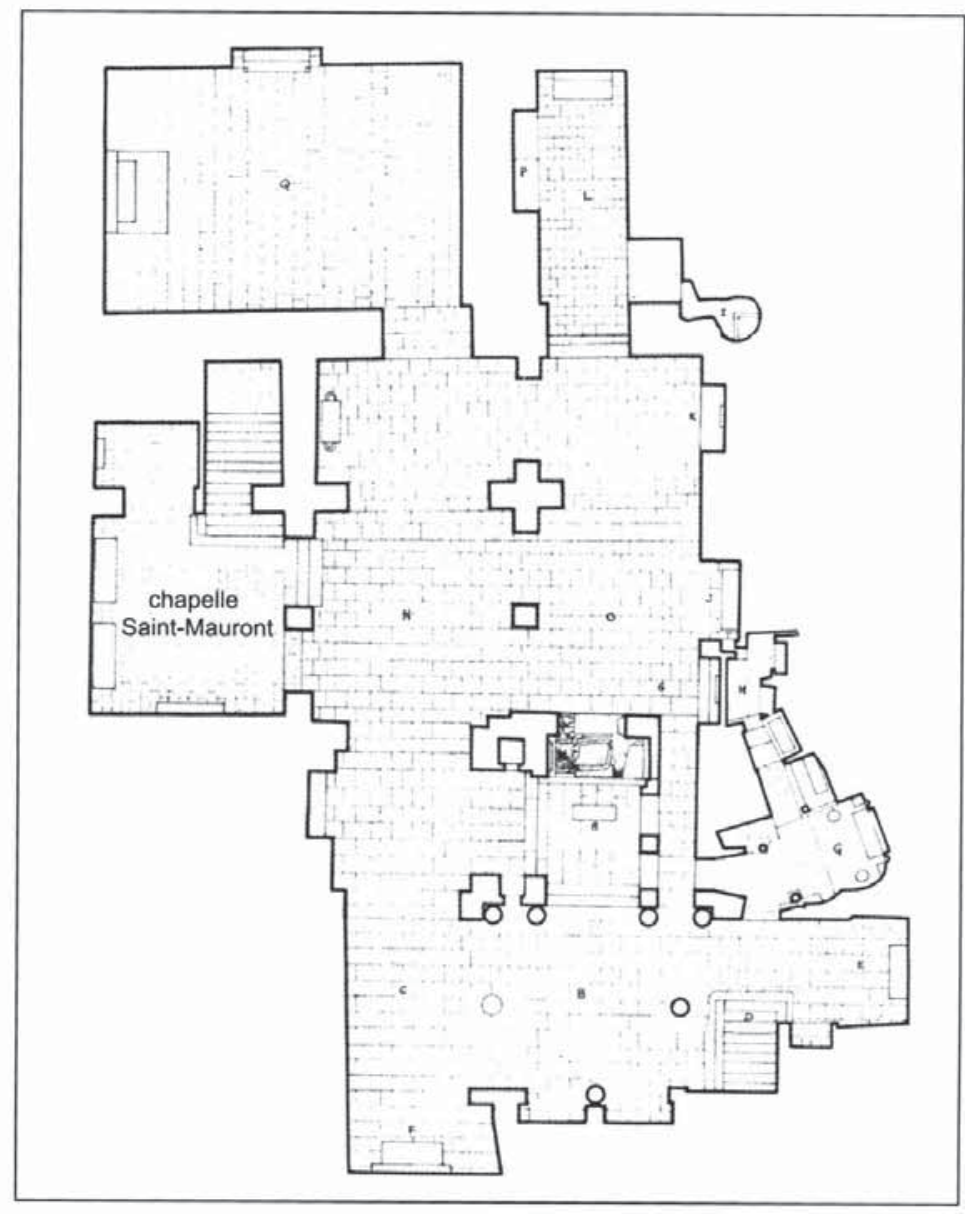

Fig. 1 - Situation de la chapelle Saint-Mauront dans les cryptes d'après le plan dressé par M. Hermite, A.C.M.H., en nov. 1968. 


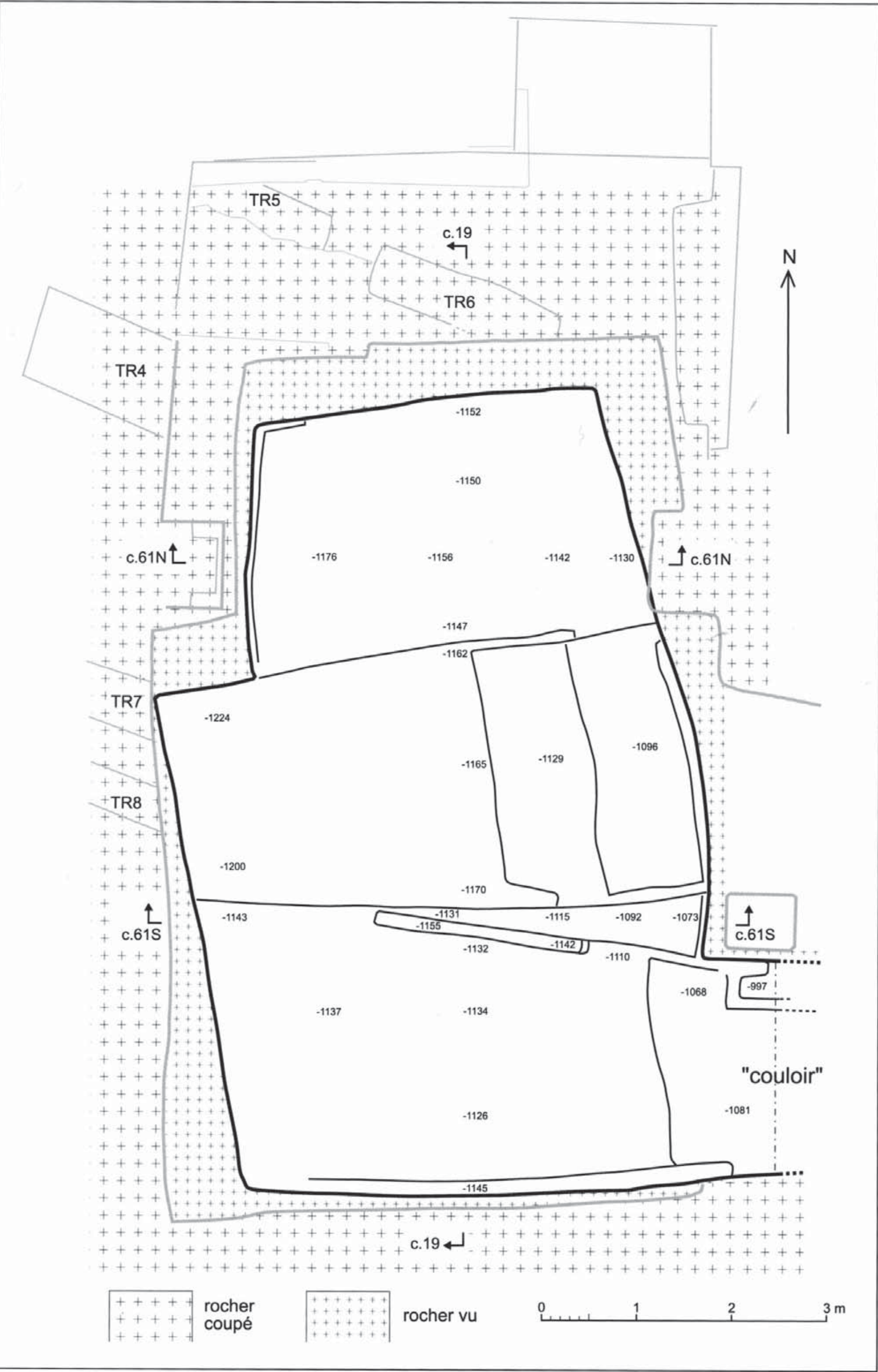

Pl. I - Le fond de la carrière. 


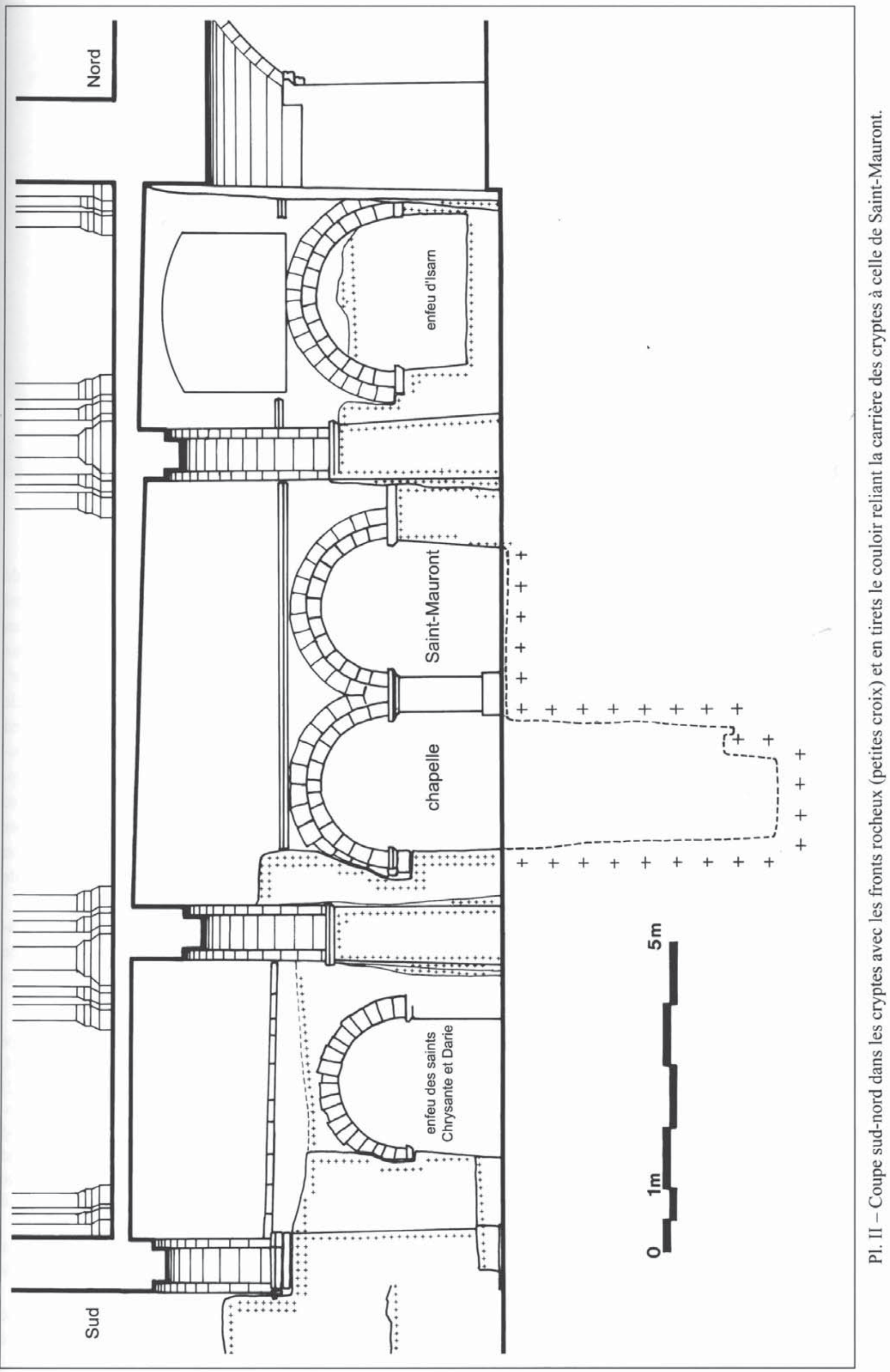




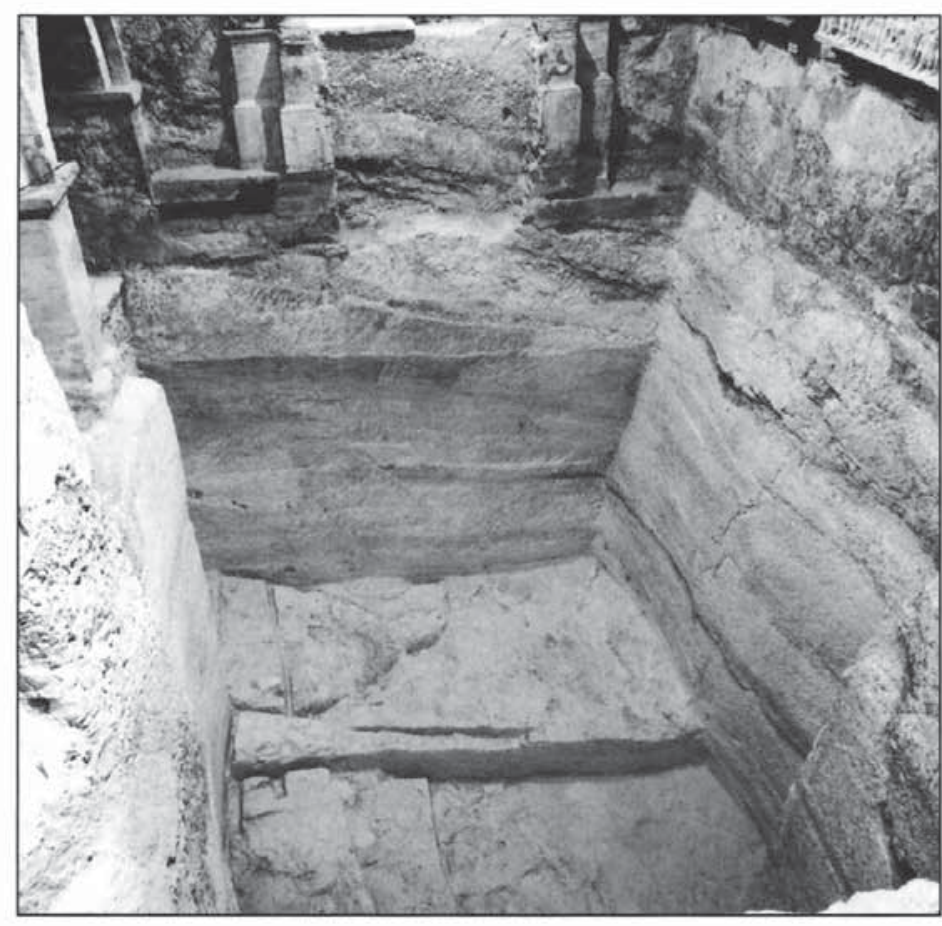

Fig. 2 - Le fond de carrière, vue vers le sud.

Les trois chantiers se distinguent par des tranches verticales sur la hauteur des parois et deux longues transversales au fond. La surface atteinte (pl. I) est plus régulière dans celui du nord, avec une pente de $11 \%$; une saignée très peu profonde longe le front de masse à l'ouest. Dans celui du centre, le plus profond, la pente est de $20 \%$; deux grands blocs longs de 2,50 et $2,70 \mathrm{~m}$ pour 0,90 à $1 \mathrm{~m}$ de largeur et $0,30 \mathrm{~m}$ de hauteur sont incomplètement détourés par des tranches larges de 0,10 à $0,15 \mathrm{~m}$ et de profondeur inégale. Dans celui du sud, le moins profond, la pente est du même ordre que celle du chantier nord, mais les surfaces laissées par la rupture des blocs y sont plus tourmentées. L'ensemble des traces conservées permet d'envisager une extraction simultanée sur les trois chantiers avec une progression d'ouest en est, l'avancement de l'exploitation dans celui du centre libérant des fronts de taille pour les deux autres au nord et au sud. Le volume ainsi excavé dans cette carrière est de $200 \mathrm{~m}^{3}$ environ (fig. 2 et 3 ).

Le comblement (pl. III, IV, V) a été effectué par l'arcature d'entrée au sud, d'abord d'est en ouest avec des blocs de rocher à l'état brut puis des déchets de taille, ensuite vers le nord jusqu'à la formation d'une pente d'équilibre de $45^{\circ}$ (couche $4 \mathrm{~b}$, assez pauvre en matériel). Puis le vide subsistant a été remblayé à travers l'arcature d'entrée nord avec des terres brunes (couche $4 \mathrm{a}$ ) provenant des niveaux anciens des alentours comme en témoignent les nombreuses céramiques antiques tardives (près d'un millier de tessons de claires D, DS.P., communes grises et amphores) associées à 13 monnaies et petits bronzes $I V^{\circ}-V^{e}$ siècle et une monnaie de Childebert I ${ }^{\text {er }}$ (511-558) ou Théodebert (534-548).

Le matériel médiéval issu de ces remblais est bien caractéristique de la période concernée. L'identification de

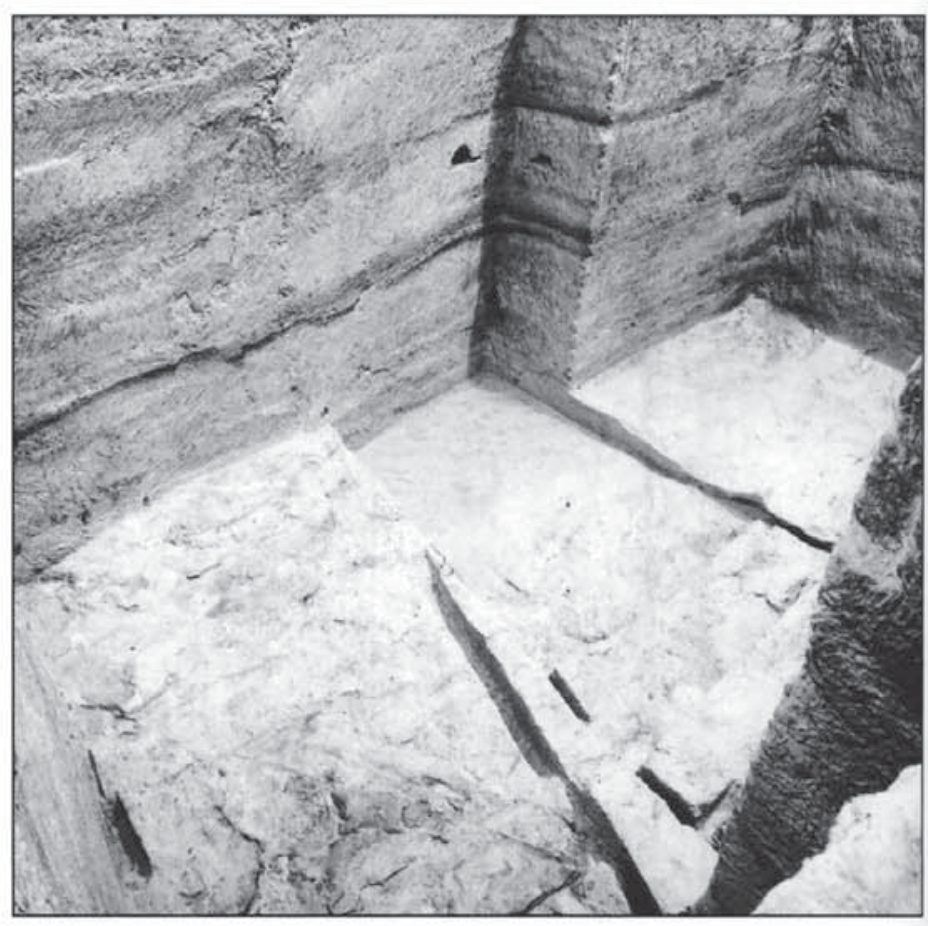

Fig. 3 - Le fond de carrière, vue vers le nord-ouest.

quatre monnaies émises entre la fin du $\mathrm{XII}^{\mathrm{e}}$ et le début du $\mathrm{XIII}^{\mathrm{e}}$ siècle ne permet pas de datation très précise ${ }^{3}$. Parmi les tessons de communes grises régionales ne figure aucun élément provenant de pégaus ou de marmites dont l'emploi est couramment attesté par ailleurs dès la fin du XII ${ }^{e}$ siècle 4. Quelques fragments sont significatifs des pots du $\mathrm{XI}^{\mathrm{e}}$ siècle et du courant du XII ${ }^{e}$ siècle : des anses rubanées, un fond bombé, un bec ponté, un rebord en poulie, un décor à la roulette. Le profil presque complet d'un récipient assez exceptionnel, peut-être résiduel lui aussi, a pu être reconstitué avec d'autres fragments retrouvés dans les remblais de la Cour ouest ${ }^{5}$. D'une contenance de l'ordre de sept litres, ce jarron qui devait comporter deux larges anses verticales présente un décor à la molette et des cordons rapportés (fig. 4).

En ce qui concerne les tessons de céramique locale glaçurée, deux lampes (fig. $5, \mathrm{n}^{\circ} 1$ et 2 ) et un fond de cruche en pâte rouge sont caractéristiques des premières produc-

3. Dans les déchets de taille $4 b$, un denier dit raimondin, $1^{\text {er type }}$ $\mathrm{XII}^{\mathrm{e}}$ ou début $\mathrm{XIII}{ }^{\mathrm{e}} \mathrm{s}$. dont la frappe a commencé avant 1184, attribuable à Raimond V (1148-1194), Raimond VI (1194-1222) ou Raimond VII (1222-1249). Dans les terres 4a, une obole coronat d'Alphonse I ou II d'Aragon (de 1181à1209) ou Raimond Bérenger V (1209-1245), un Royal dit denier à la mitre, Raimond Bérenger IV (1172-1181) ou Alphonse I ${ }^{\text {er }}$ (1181-1196) en pariage avec l'archevêque d'Arles (1177-1186), et une obole de Melgueil $\left(\mathrm{XI}^{\mathrm{e}}-\mathrm{XIII} \mathrm{e}^{\mathrm{s}} \mathrm{s}.\right)$.

4. J.-P. Pelletier, Les céramiques communes régionales en pâtes brunes et grises, in Marchesi et al., Marseille, les ateliers de potiers, p. 66-76.

5. Ce qui confirme que le remblaiement de la carrière a été effectué avec des terres provenant de l'extérieur de l'église, à proximité immédiate. 


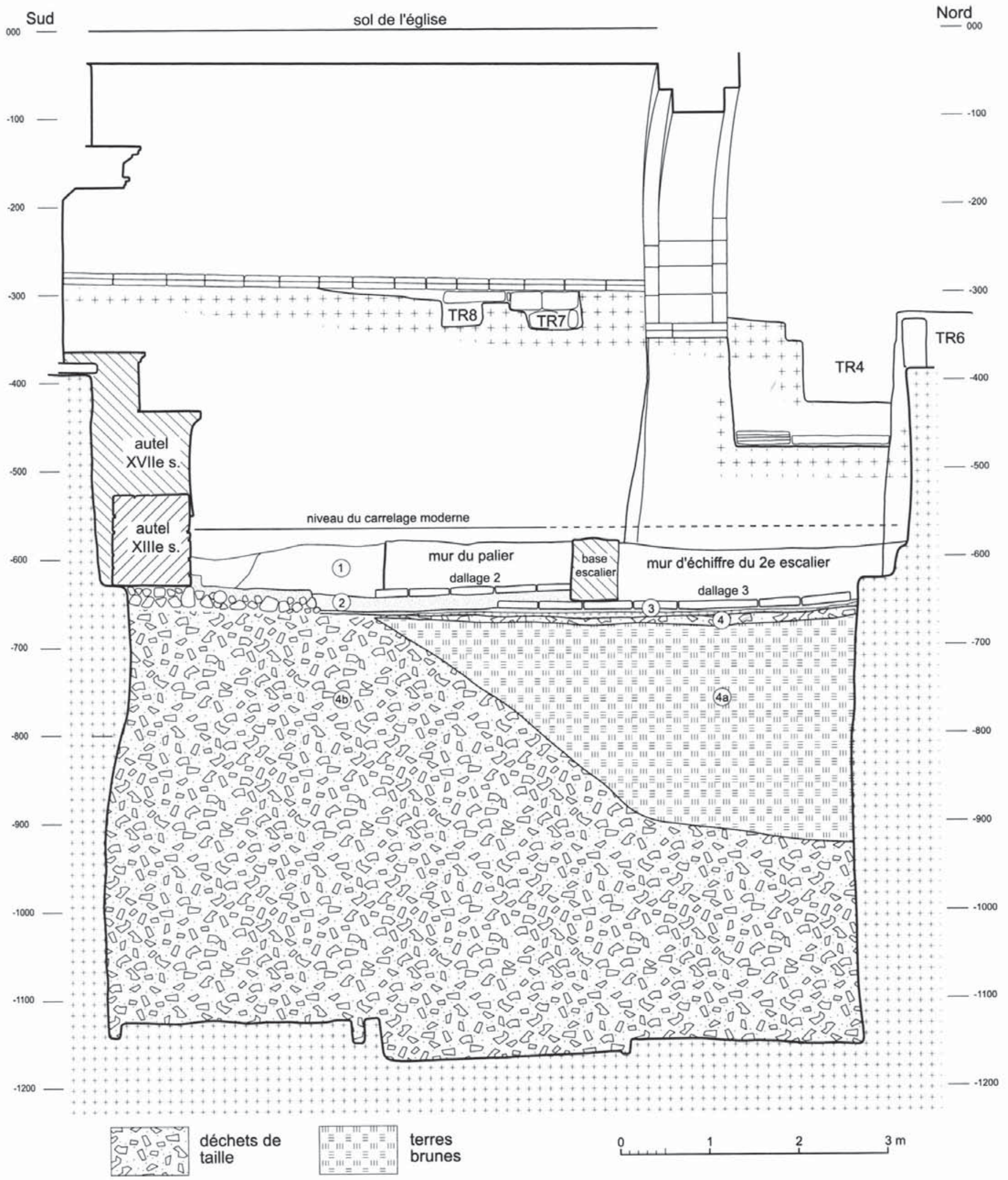

PI. III - Coupe sud-nord 19. 


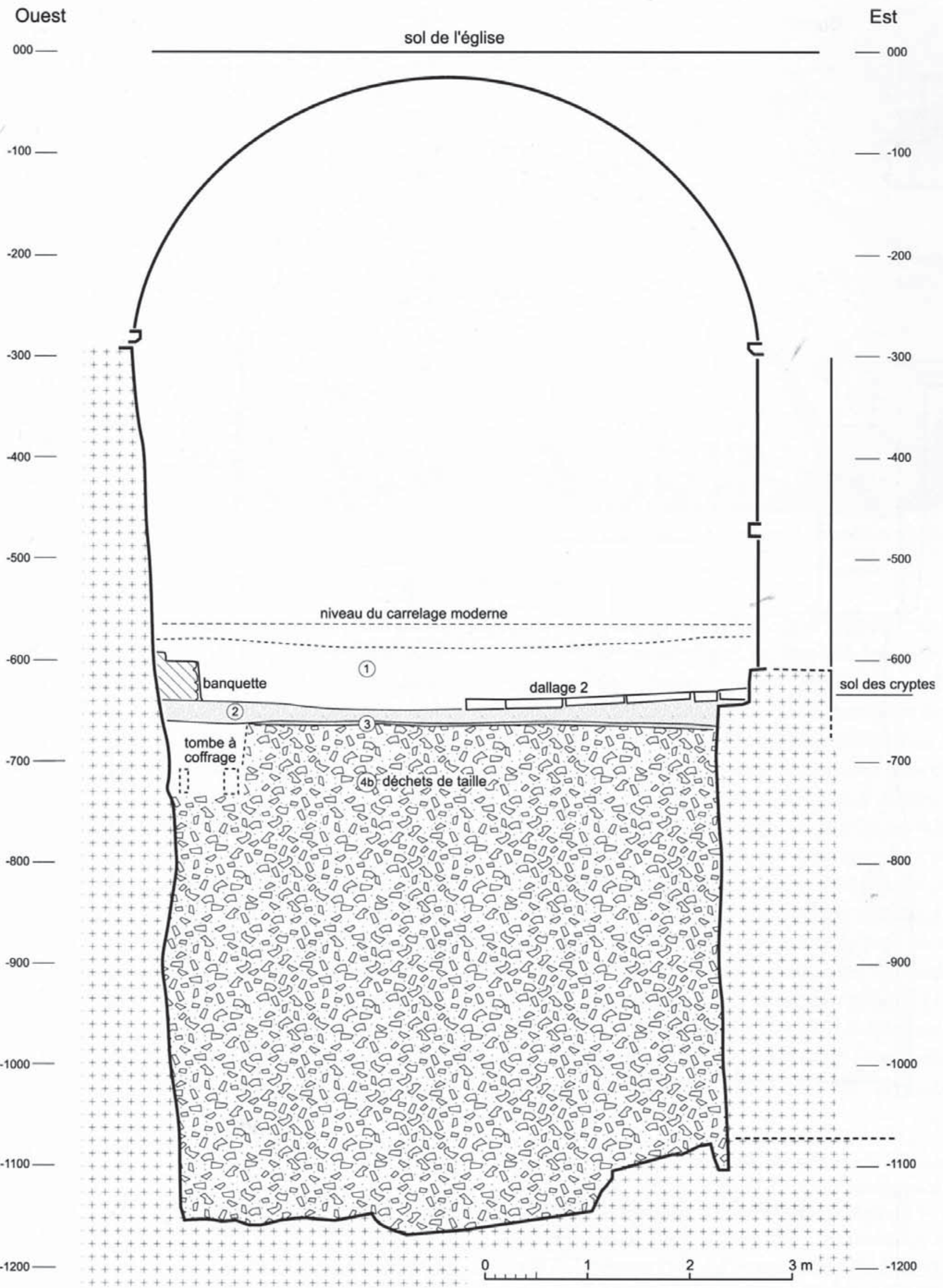


Ouest

Est

$000-$

$-100$

.200

300

$600-$

$-700$

\begin{tabular}{l|l} 
& niveau du carrelage moderne \\
&
\end{tabular}

1
$1+++++$
+++++
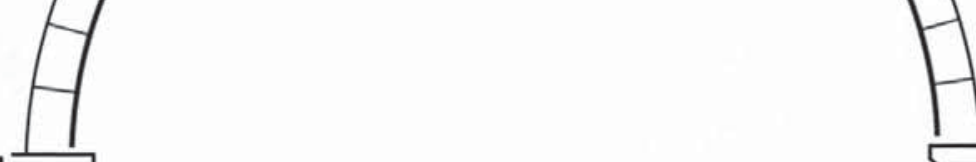

$-400$

$+++$

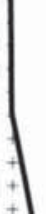

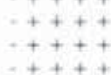

$+++++$

$-.500$
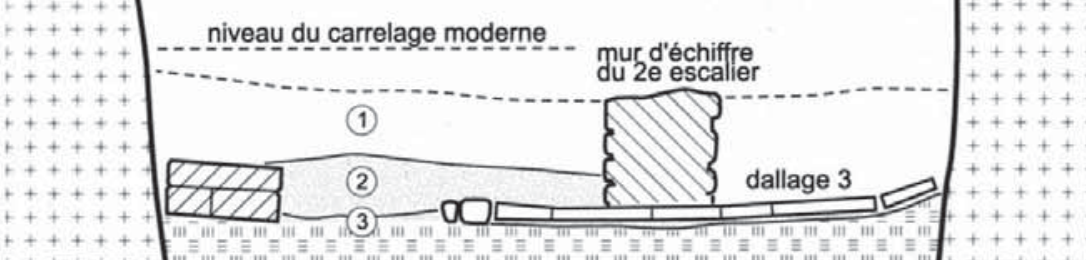

$-600$

$-700$

sépulture

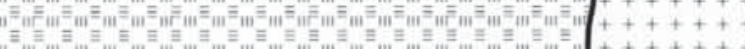

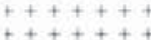

$t+t+++1 \equiv$

$+++++++$

$++++++++$

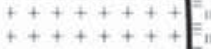

(it)

$++++++$

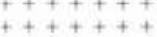

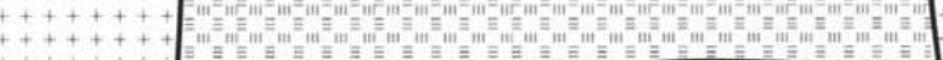

a

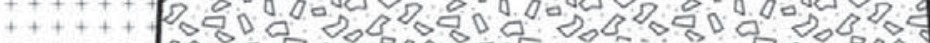

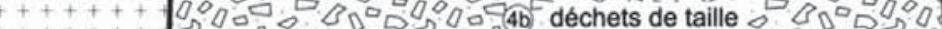

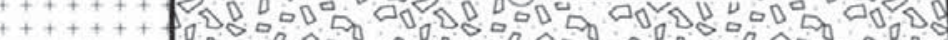

+
+++++++
+++++++
+

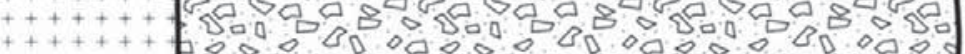

$-1000$

$-1000$

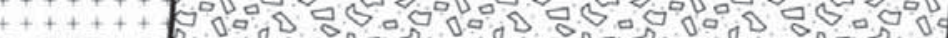

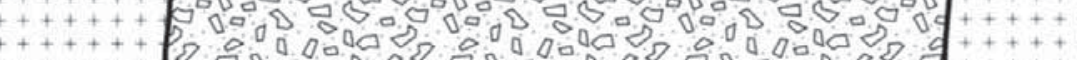

+++++++0 皮

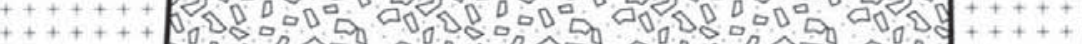

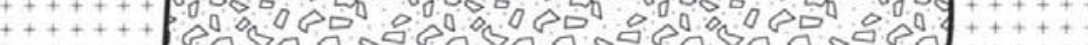




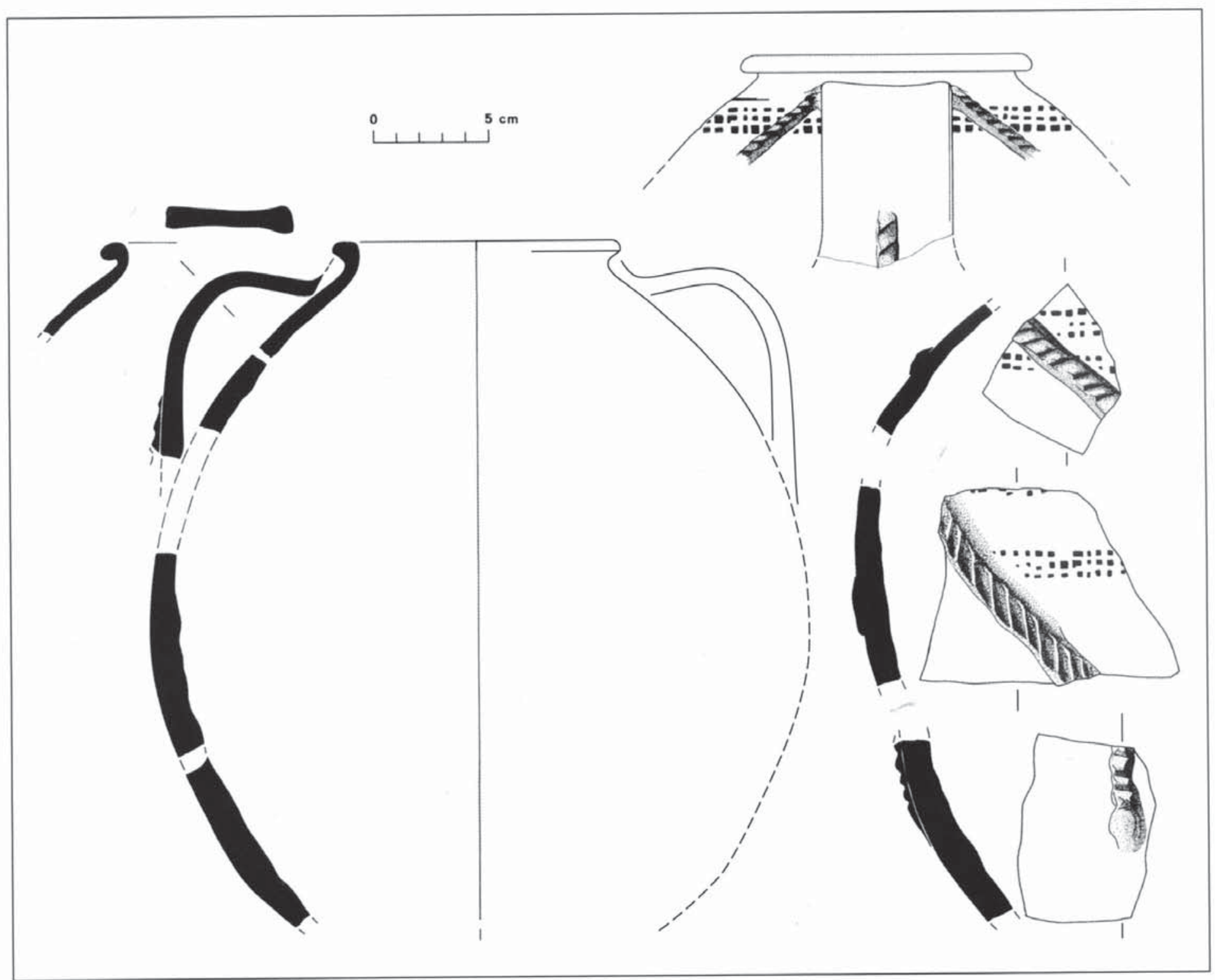

Fig. 4 - Jarron en céramique commune grise (XII ${ }^{\mathrm{e}}$ siècle).

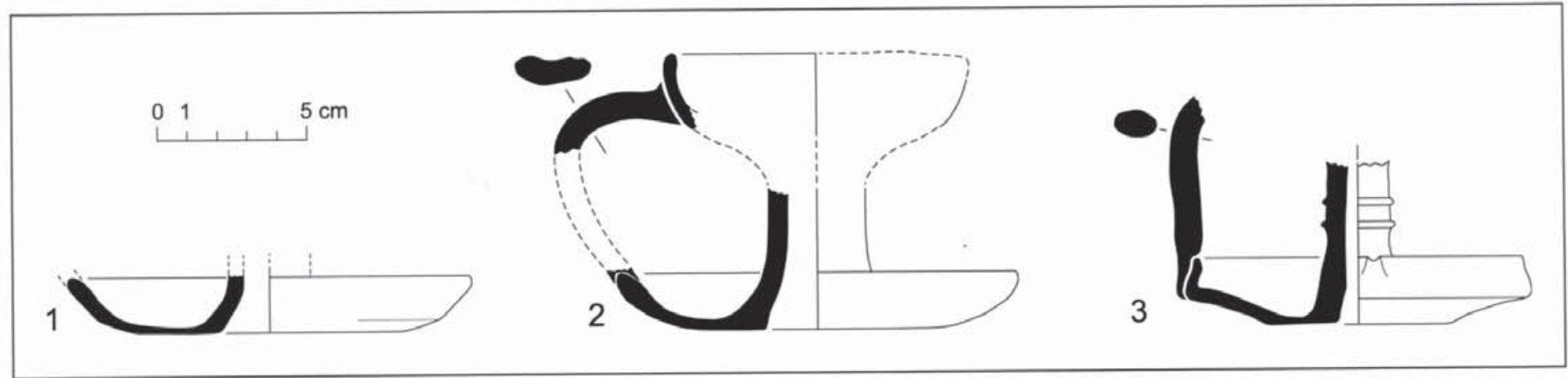

Fig. $5-\mathrm{n}^{\circ} 1$ et 2 : lampes en pâte rouge glaçurée, atelier de Marseille Sainte-Barbe (début XIII ${ }^{\circ}$ siècle); $\mathrm{n}^{\circ} 3$ : lampe à émail monochrome turquoise d'origine valencienne (début XIII' ${ }^{\mathrm{e}}$ siècle). 
tions des ateliers de potiers du burgus oleriorum, implanté au nord-est du rempart médiéval ${ }^{6}$. Les importations sont représentées d'abord par une belle lampe sur pied à émail monochrome turquoise d'origine valencienne (fig. $\left.5 n^{\circ} 3\right)^{7}$, dont le faciès est encore très islamisant ${ }^{8}$. S'y ajoutent un fond de jatte culinaire modelée en pâte rouge glaçurée dont la provenance ligure est aujourd'hui remise en question et un bord de coupelle à marli à glaçure verte attribué à l'aire siculomaghrébine'. L'ensemble des données fournies par les céramiques locales et importées ainsi que les monnaies conforte une datation du début du XIII ${ }^{e}$ siècle qui doit être rapprochée de la consécration de la chapelle Saint-Maurice en $1219^{10}$.

\section{B - LE PREMIER AMÉNAGEMENT DE LA CHAPELlE}

Au nord, la surface des remblais 4a fut égalisée par l'apport de 15 à $20 \mathrm{~cm}$ de déchets de taille et de terre pour la mise en place à la cote $-655 /-660 \mathrm{du}$ lit de pose d'un premier dallage de pierre (couche 3, pl. III, IV, V). Il faut attribuer à cette séquence l'inhumation d'un adulte dont le corps a été déposé à la cote -730 dans les terres $4 a$, près de la paroi nord-ouest, suivant une direction méridienne avec la tête au sud. L'emplacement fut matérialisé par un massif de deux assises maçonnées, large de 0,50 pour $2,70 \mathrm{~m}$ de long, et haut de $0,30 \mathrm{~m}$ (pl. V et VI).

Cette tombe a été recouverte par un premier escalier large de $2 \mathrm{~m}$ avec une volée basse longue de $4 \mathrm{~m}$ depuis le sud jusqu'au replat rocheux à la cote -320 au nord. Les restes de la maçonnerie du mur d'échiffre limitent le dallage conservé à la cote $-650 /-640$ à l'est, et au sud les restes de la première marche marquent son emplacement à la cote -660 . Pour déboucher dans l'axe des piles entre la première et la seconde travée de l'église, on pourrait restituer une volée continue relativement raide, tournant à angle droit vers l'est, sans palier, avec environ 33 marches profondes de $30 \mathrm{~cm}$ pour $20 \mathrm{~cm}$ de haut.

6. L. Vallauri, M. Leenhardt, Les productions céramiques, in Marchesi et al., Marseille, les ateliers de potiers, p. 266, fig. 232 et p. 216, fig. 190.

7. Voir aussi Fixot, Pelletier, Étude archéologique, fig. XXIII, $\mathrm{n}^{\circ} 1$.

8. G. Démians d'Archimbaud, C. Lemoine, Les importations valenciennes et andalouses en France méditerranéenne : essai de classification en laboratoire, in G. Démians d'Archimbaud, M. Picon (dir.), La céramique médiévale en Méditerranée occidentale, $X^{c}-X V^{e}$ siècles (Valbonne 1978), Paris, 1980, p. 359372 , ici p. 370 , pl. VIII, n 1 ; M. Mesquida Garcia, J.-E. Lopez Periz, S. Prades, R. Smolka, Las ollerias de Paterna Tecnologia y produccion, volumen I siglos XII y XIII, Paterna, 2001, ici p. $385, \mathrm{n}^{\circ} 12$.

9. C. Capelli, F. Parent, C. Richarte, L. Vallauri, R. Cabella, Ceramiche invetriate di importazione in Provenza in epoca bassomedievale : Dati archeologi e archeometri, in Atti XXXVII - XXXVIII Convegno internazionale della ceramica 2004-2005, Savona, 2006, p. 189-200.

10. Voir Fixot, Pelletier, Étude archéologique, IX, n. 174.

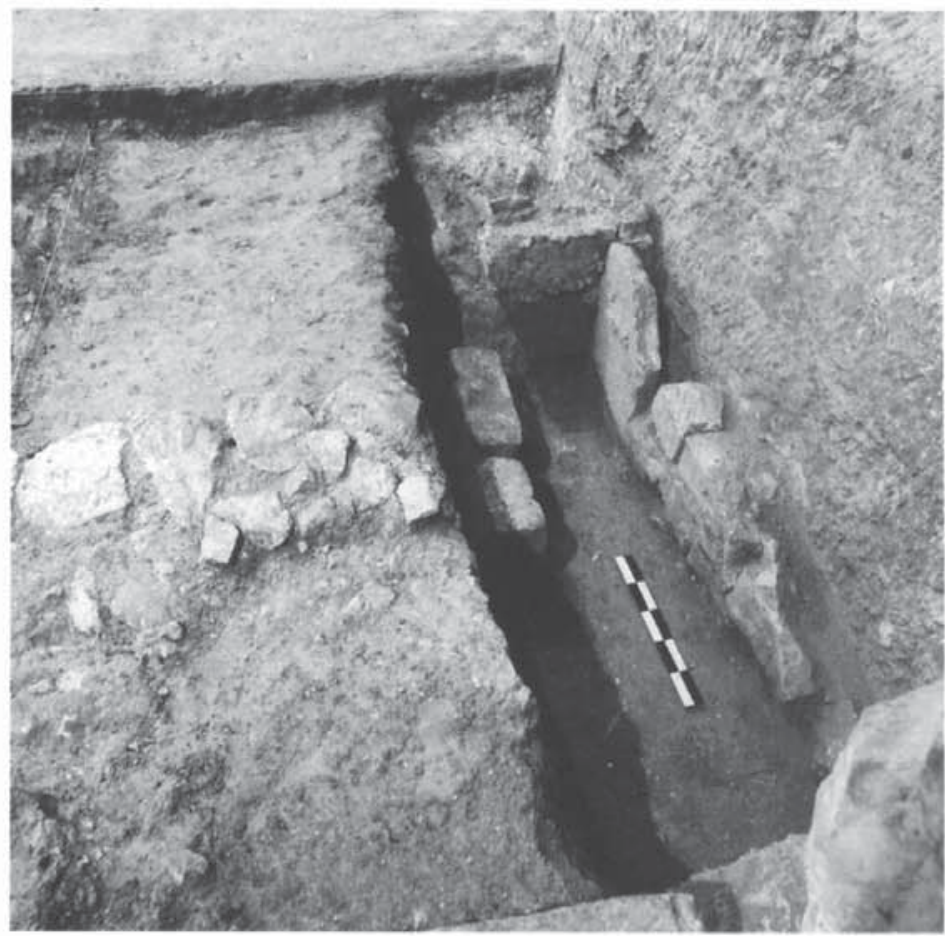

Fig. 6 - La base du mur de l'escalier primitif coupée par la fosse de la tombe à coffrage, vue vers le sud.

Il peut paraître curieux que la tombe ait été masquée par cet escalier maçonné. Son massif de couverture est posé directement sur les terres $4 \mathrm{a}$, comme les restes de maçonnerie du blocage de l'escalier qui viennent buter contre lui. D'autre part, ce massif, dont l'arase est à la cote -628 , à $20 \mathrm{~cm}$ au-dessus du niveau du dallage primitif, a été aussi recouvert par les remblais supportant un deuxième dallage disparu à cet endroit mais qui se trouvait à ce même niveau. Le signalement de cette sépulture n'a donc jamais été visible bien longtemps, tout au plus sous un escalier provisoire en bois?

Contre la paroi méridionale, sur les déchets de taille, l'autel et son podium ont été bâtis sur un hérisson de pierres (pl. III) qui contenait une obole marseillaise frappée après 1257 (Charles Ier, jusqu'en 1285, ou Charles II d'Anjou, $1285-1309)^{11}$. Le podium, à la cote $-630 /-635$ autour de l'autel, devait être surélevé d'une vingtaine de $\mathrm{cm}$, soit la hauteur d'une marche, par rapport au dallage primitif coté à -650 au nord, dans le reste de la chapelle. Depuis les cryptes, l'entrée s'effectuait de part et d'autre du pilier maçonné supportant les arcatures géminées.

\section{C - Le deuxiĖme AMÉnAgEMENT DE LA CHAPELlE}

À l'ouest, une seconde sépulture, à coffrage, a été vidée et partiellement détruite au début de l'époque moderne. La fosse, creusée dans les déchets de taille, avait coupé les maçonneries de la base du premier escalier ainsi que le mortier de pose du premier dallage (fig. 6 et pl. VI). Sa mise

11. Cette datation, nettement plus tardive que celle de la consécration de la chapelle et du matériel inclus dans les remblais, implique sans doute une réfection de l'autel. 


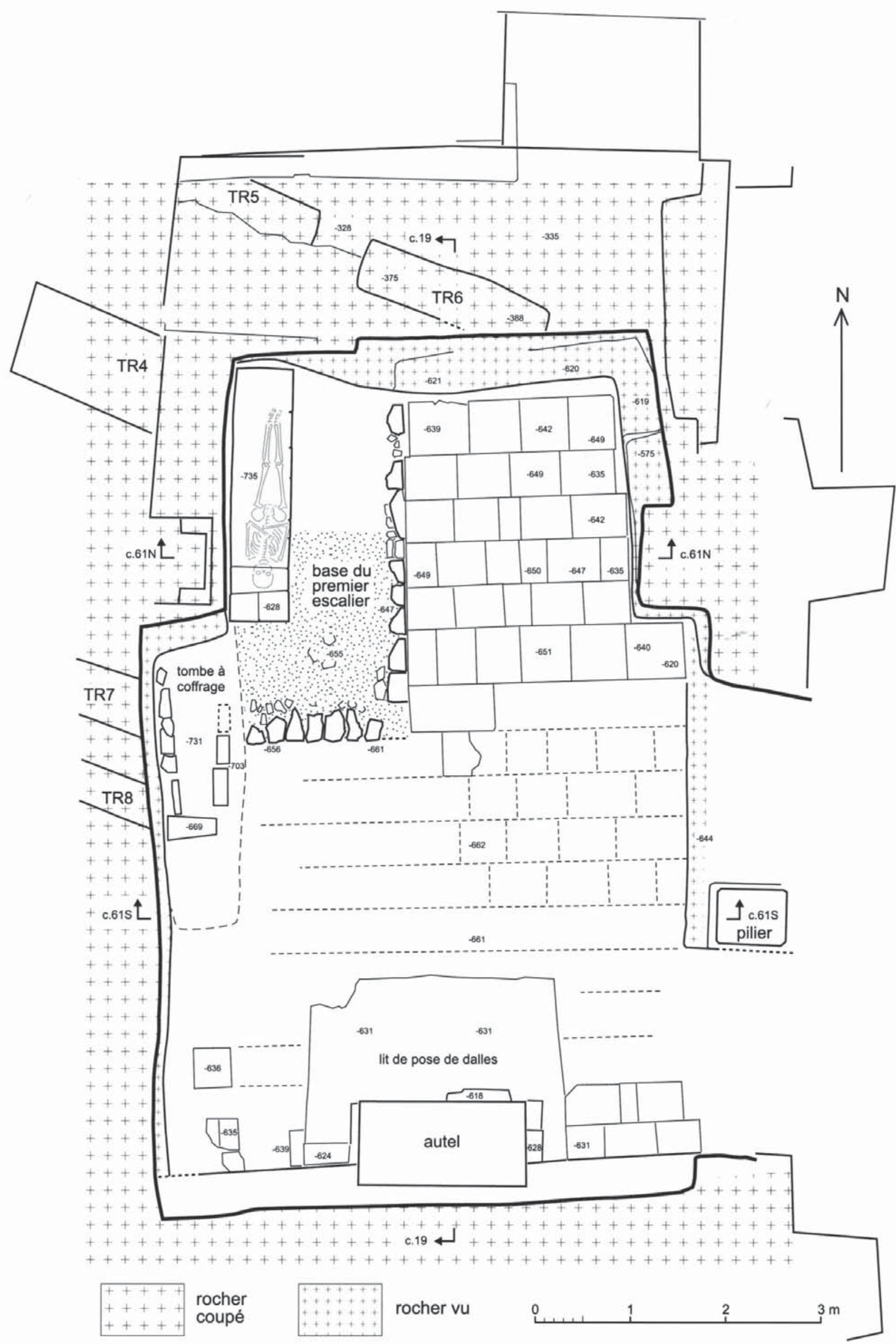

Pl. VI - Le premier état de la chapelle (XIII ${ }^{e}$ siècle). 
en place se situe donc au cours d'une deuxième séquence de travaux, sans doute étalée dans le temps.

Après le démontage du premier escalier, ces réfections sont marquées d'abord par l'exhaussement du sol avec un nouveau dallage de pierre établi à la cote -630 environ soit au même niveau que celui qui se trouvait de part et d'autre de l'autel et dans les cryptes, ensuite par la construction d'une longue banquette le long de la paroi occidentale, et surtout par un nouvel escalier plus praticable. Longeant le mur occidental de l'église, une première volée descendait vers le nord jusqu'à un palier que quelques marches séparaient d'un autre palier à l'est, avant une série de degrés vers le sud (fig. 7 et pl. VII).

Par la suite, la base de l'escalier fut prolongée par un palier surélevé d'une trentaine de $\mathrm{cm}$ qui débouchait directement vers les cryptes par l'arcature nord et quatre marches. Ainsi l'espace dévolu à la chapelle était distinct de celui réservé au passage (fig. 9 et pl. VIII).

Les remblais supportant le nouveau sol (couche 2, pl. III, IV V et fig. 6,7 ) ont fourni trois monnaies : un liard au dauphin de François I ${ }^{\text {er }}$ (1544-1547), un liard de Charles IX (1574) et un patac de billon de Grégoire XIII (fin XVI ${ }^{\mathrm{c}}$ siècle); un liard à l' $F$ de François ${ }^{\mathrm{er}}$ a été découvert dans la maçonnerie de la banquette. Outre un rebord émaillé et peint en brun de coupe tunisienne du XIII ${ }^{e}$ siècle et des faïences espagnoles à décor vert et brun de Valence et de Barcelone de la première moitié du XIV $V^{\bullet}$ siècle dont le caractère résiduel est évident, la présence dans cette couche d'écuelles valenciennes émaillées et décorées en bleu et lustre métallique (fig. 8) correspond bien aux données monétaires ${ }^{12}$.

\section{D - LeS RÉAMÉNAGEMENTS DU XVII ${ }^{E}$ SIĖCLE $^{13}$}

Reprenant le plan de l'escalier précédent, ainsi qu'un certain nombre de ses éléments dont témoignaient des marches usées sur les deux faces, la nouvelle descente vers les cryptes aboutissait au niveau du palier inférieur déjà évoqué. Le second mur d'échiffre reposait directement sur le dallage primitif( $\mathrm{pl}$. III). L'espace fut réunifié par la pose à la cote -565 environ d'un troisième sol en carreaux de terre cuite de $20 \times 20 \mathrm{~cm}$. Le bouchage de la plus méridionale des arcatures géminées isolait des cryptes la chapelle proprement dite, le seul passage se faisant désormais par celle du nord avec quatre marches comme précédemment (pl. IX).

Masquant complètement la banquette et une partie du support de l'autel médiéval, sous le carrelage, l'épaisse couche 1 de remblais $(60$ à $70 \mathrm{~cm})$, terres noirâtres provenant d'un

12. D. Carru (dir), De l'Orient à la table du Pape. L'importation des céramiques méditerranéenes dans la région d'Avignon aux $X I V^{-}-X V T^{e}$ siècles, in Documents d'archéologie vauclusienne, 5 , Avignon 1995, ici p. 60-76.

13. Ces transformations doivent être associées à celles observées dans le reste du monument, notamment dans le « Porche d'Isarn » : Cf. Fixot, Pelletier, Étude archéologique, IX H 4 et 5.

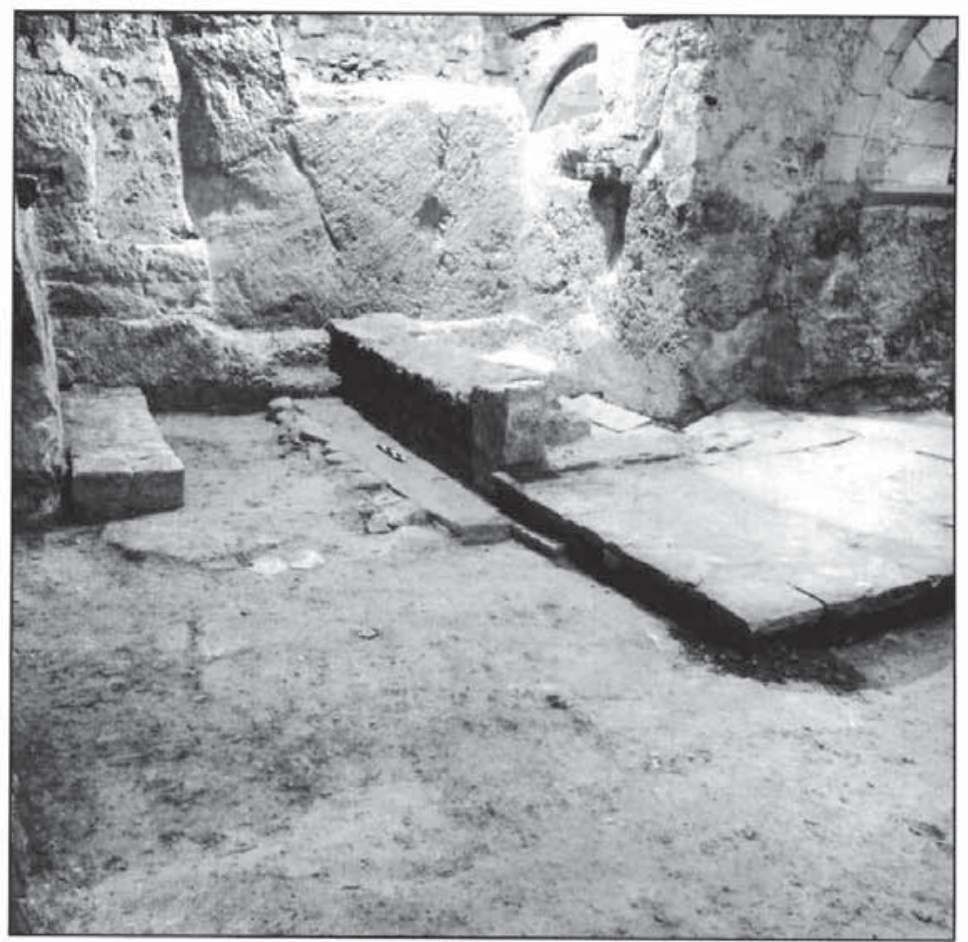

Fig. 7-Au centre le mur d'échiffre sur le premier dallage, le second dallage ; à gauche le massif maçonné, souvenir de la sépulture (?), vue vers le nord.

dépotoir, a fourni un matériel abondant. Parmi les dix-sept monnaies ${ }^{14}$ retrouvées, la plus tardive est un double tournois de Louis XIII de 1643.

Près de 7000 tessons ${ }^{15}$ témoignent des céramiques alors en usage dans l'abbaye. En dépit de la fragmentation, on peut estimer qu'ils provenaient de 190 objets au minimum, parmi lesquels figurent des pipes, des jarres, et un nombre impressionnant de vaisselles de table (110 objets) et de poteries culinaires ( 80 objets). Leur origine est très variée mais l'Italie figure en bonne place. Les faïences toscanes unies ou décorées voisinent avec celles ligures à décor bleu inspiré de la Chine ou de la Turquie ${ }^{16}$. Plus exceptionnels sont les quelques exemplaires polychromes rehaussés d'or venus d'Iznik et du Proche-Orient ; leur présence évoquée

14. Onze double tournois de Louis XIII datés $1624,1632,1633$, $1635,1639,1640,1642,1643$; un double tournois de Frédéric Henri (Orange), 1642 ; deux double tournois de Gaston d'Orléans (Dombes), 1639 ; un douzain aux croissants de Henri II (1547-1559) ; un douzain de Charles X roi de la Ligue, 1590, contremarqué d'un lys sous Louis XIII ; un jeton de Nüremberg, fin $\mathrm{XVI}^{\mathrm{e}}$-début $\mathrm{XVII}{ }^{\mathrm{e}} \mathrm{s}$.

15. Comptage effectué par V. Abel ; voir V. Abel, G. Démians d'Archimbaud, L'humilité selon Saint-Victor, in Un goût d'Italie : céramiques et céramistes italiens en Provence du Moyen Âge au $X X^{c}$ siècle; V. Abel, H. Amouric (dir.), Catalogue de l'exposition, Aubagne 1993, Aix-en-Provence, 1993, p. 74-75.

16. N. Atasoy, J. Raby, Iznik. La poterie en Turquie ottomane, éd. Y. Petsopoulos, Chêne, 1996 ; L. Pessa (L.) (dir.), Le ceramiche liguri, Milano, 2005 ; illustrations in Fixot, Pelletier, Saint-Victor de Marseille, 1, Étude archéologique et monumentale, fig. XXIII, $n^{\circ} 2,3,4$. 

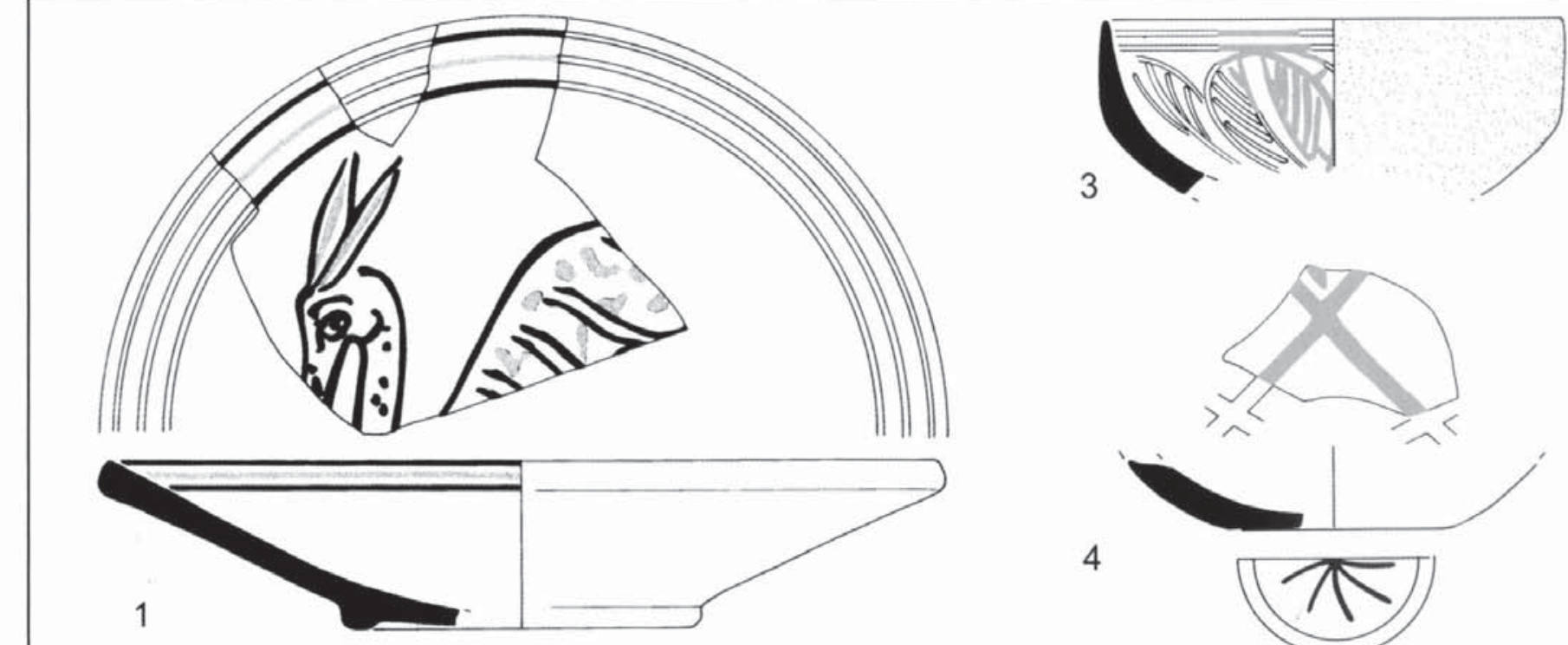

3

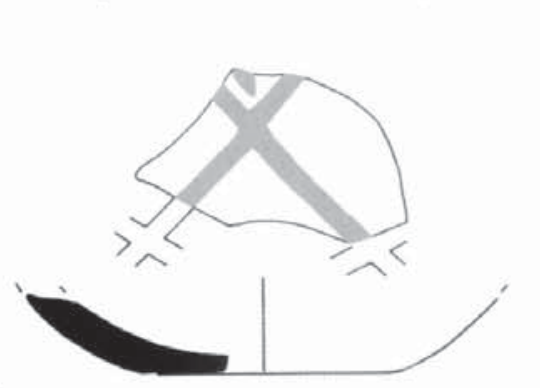

4
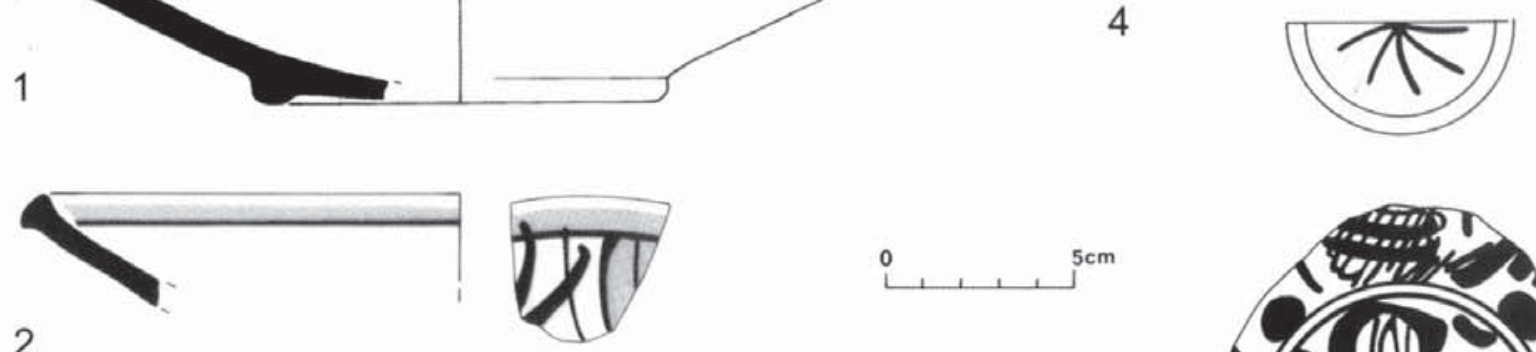

2

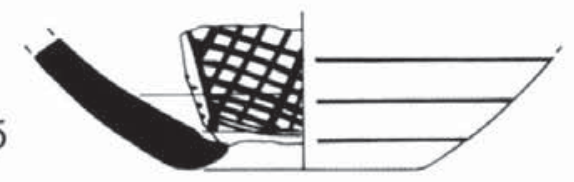

6

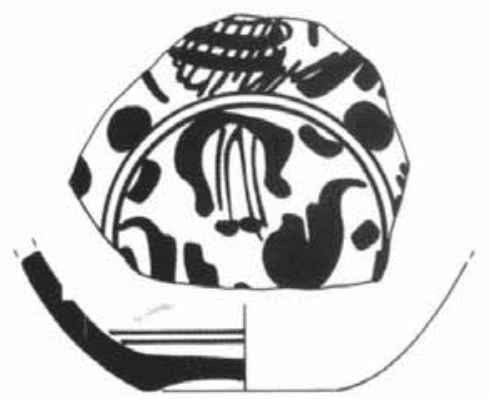

Fig. 8 - Vaisselles décorées provenant des couches 2. Coupes à décor vert et brun sur émail (première moitié du XIV siècle) d'origine catalane $\left(n^{\circ} 1\right)$ et valencienne $\left(n^{\circ} 2\right)$; écuelles émaillées de Valence $\left(X V^{\bullet}\right.$ siècle) à décor bleu $\left(n^{\circ} 3\right)$, bleu et lustre métallique $\left(n^{\circ} 4\right)$; $\mathrm{n}^{\circ} 5$ et 6 : écuelles émaillées à décor de lustre métallique, Valence $\left(\mathrm{XVI}^{\mathrm{e}} \mathrm{s}\right)$.

dans les textes a été rarement illustrée par des découvertes archéologiques à l'exception de quelques tessons marseillais $^{17}$. Mais le service le mieux représenté se compose d'assiettes et bols pisans, à décor d'engobes marbrés, ou à décor incisé avec un œillet ou blason très stéréotypés. Ces céramiques " graffita tarda », colorées, attrayantes et fines, rivalisent avec les productions régionales d'Apt, de Fréjus et de la vallée de l'Huveaune ${ }^{18}$. Quant aux poteries culinaires, elles proviennent de Biot en Provence orientale ${ }^{19}$, de Catalogne et de Ligurie. Une série importante de marmites en pâte grise, dont l'origine reste toujours indéterminée, a été pour la première fois mise en évidence (fig. 10). Cette production archaïque, cuite en atmosphère réductrice, est aujourd'hui attestée dès le milieu du XVI ${ }^{\mathrm{e}}$ siècle et encore au XVII ${ }^{e}$ siècle Elle a connu une ample diffusion depuis la

17. Amouric et al., Vingt mille pots, p. 112-116; illustrations in Fixot, Pelletier, Saint-Victor de Marseille, 1, Étude archéologique et monumentale, fig. XXII.

18. Amouric et al., Vingt mille pots, p. 79 et 91 ; illustrations in Fixot, Pelletier, Saint-Victor de Marseille, 1, Étude archéologique et monumentale, fig. XXIII, ${ }^{\circ} 5$ et 6.

19. H. Amouric, L. Argueyrolles, L.Vallauri, Biot, Jarres, terrailles et fontaines, $X V I^{e}-X X^{e}$ siècles, Arezzo, Biot, 2006, ici p. 70-73.

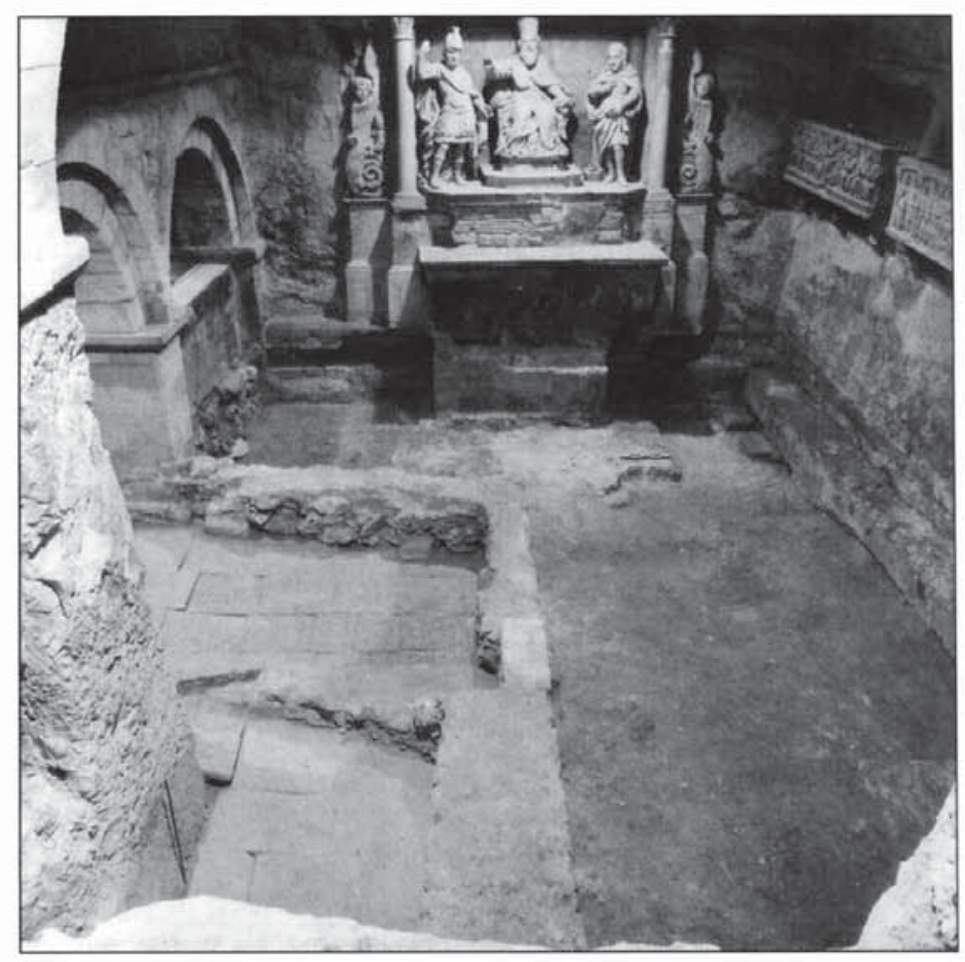

Fig. 9 -Au $1^{\text {er }}$ plan, le mur d'échiffre et celui du palier bâtis sur le premier dallage ; à l'arrière-plan, les trois statues, l'autel médiéval sous celui du XVII ${ }^{\mathrm{e}}$ siècle, et à gauche le bouchage de l'arcature sud (vue vers le sud). 


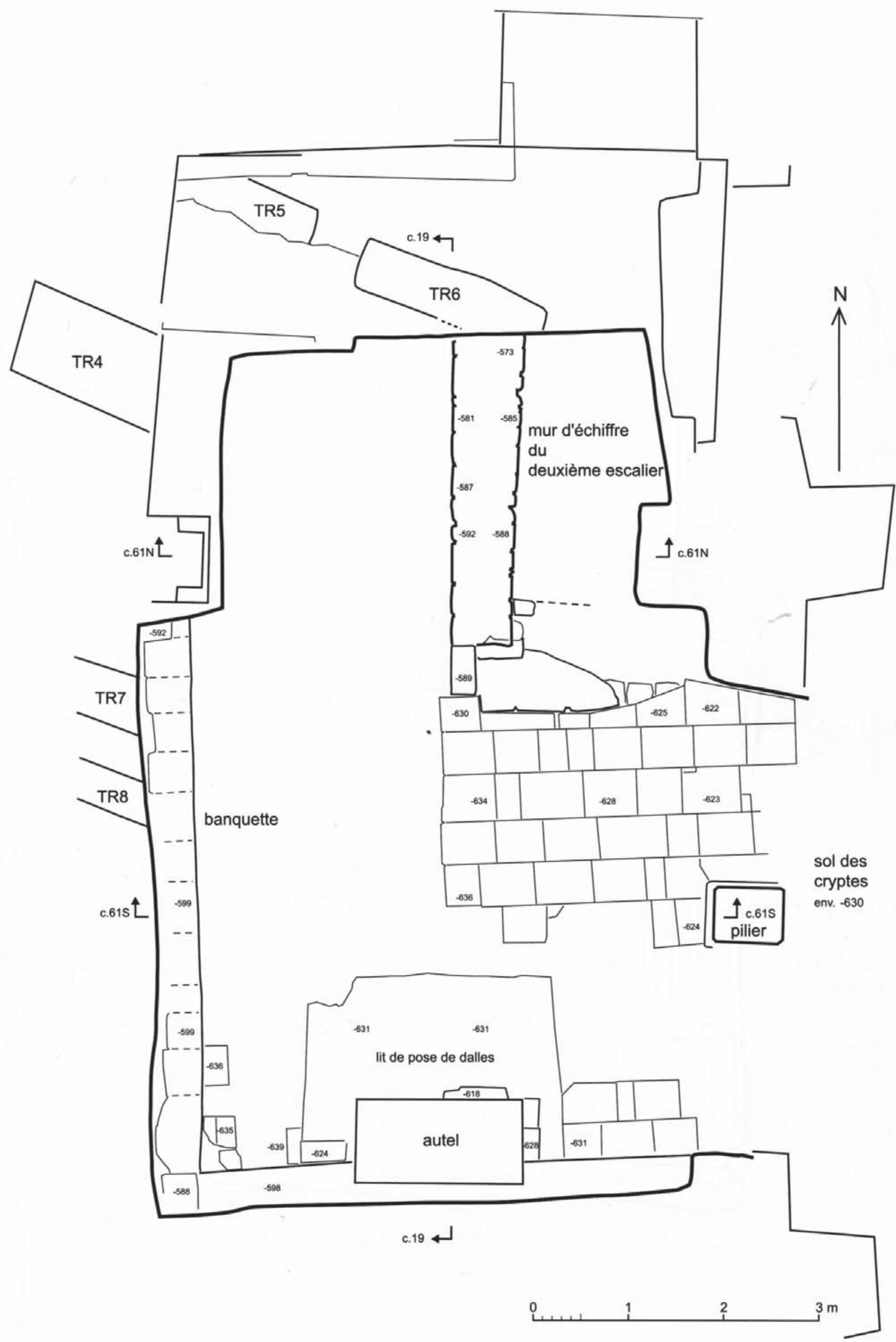

$\mathrm{Pl}$. VII - Le deuxième état de la chapelle (XVI ${ }^{\mathrm{e}}$ siècle). 


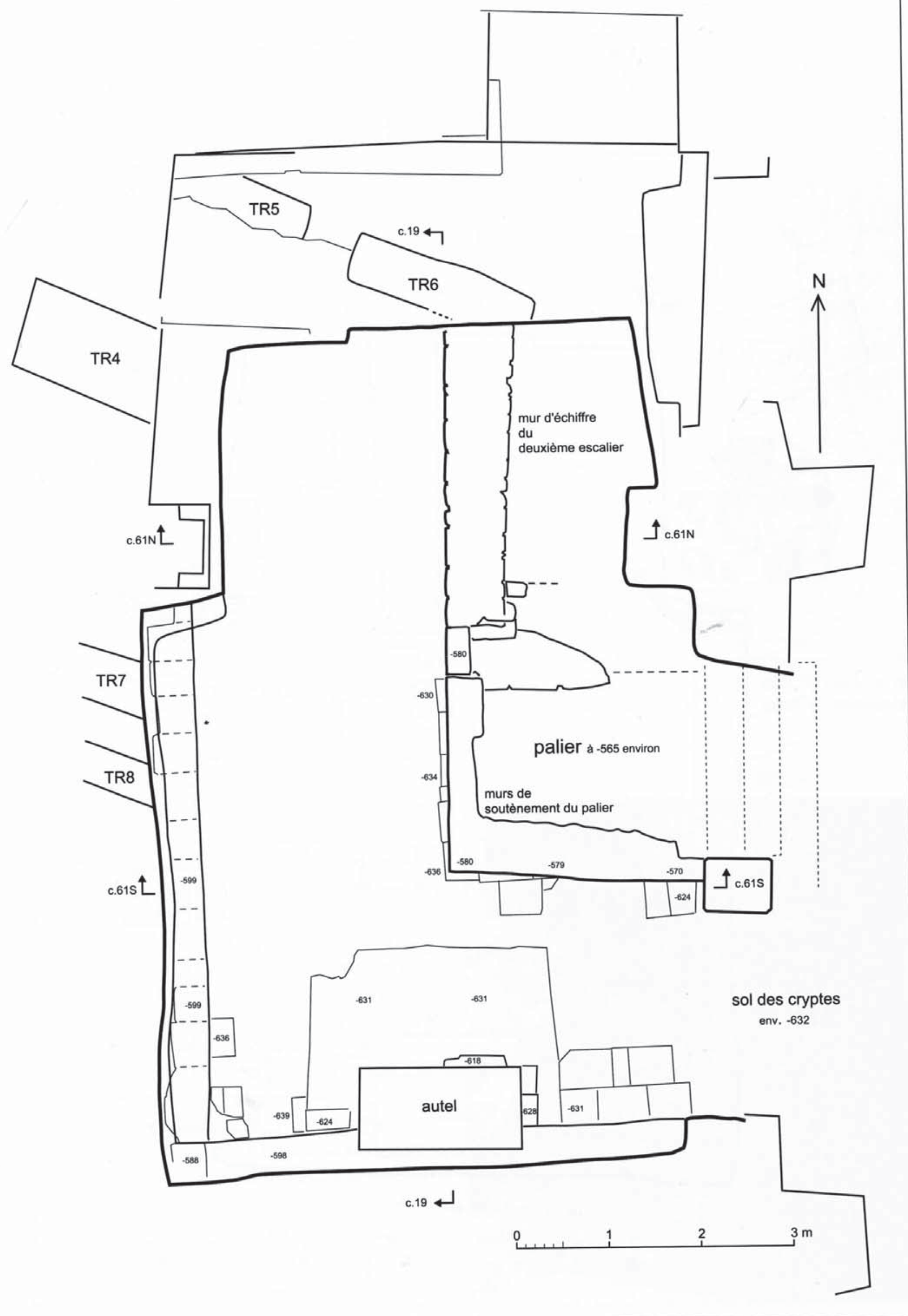

Pl. VIII - Le troisième état de la chapelle $\left(\mathrm{XVI}^{\mathrm{e}}\right.$ siècle $)$. 


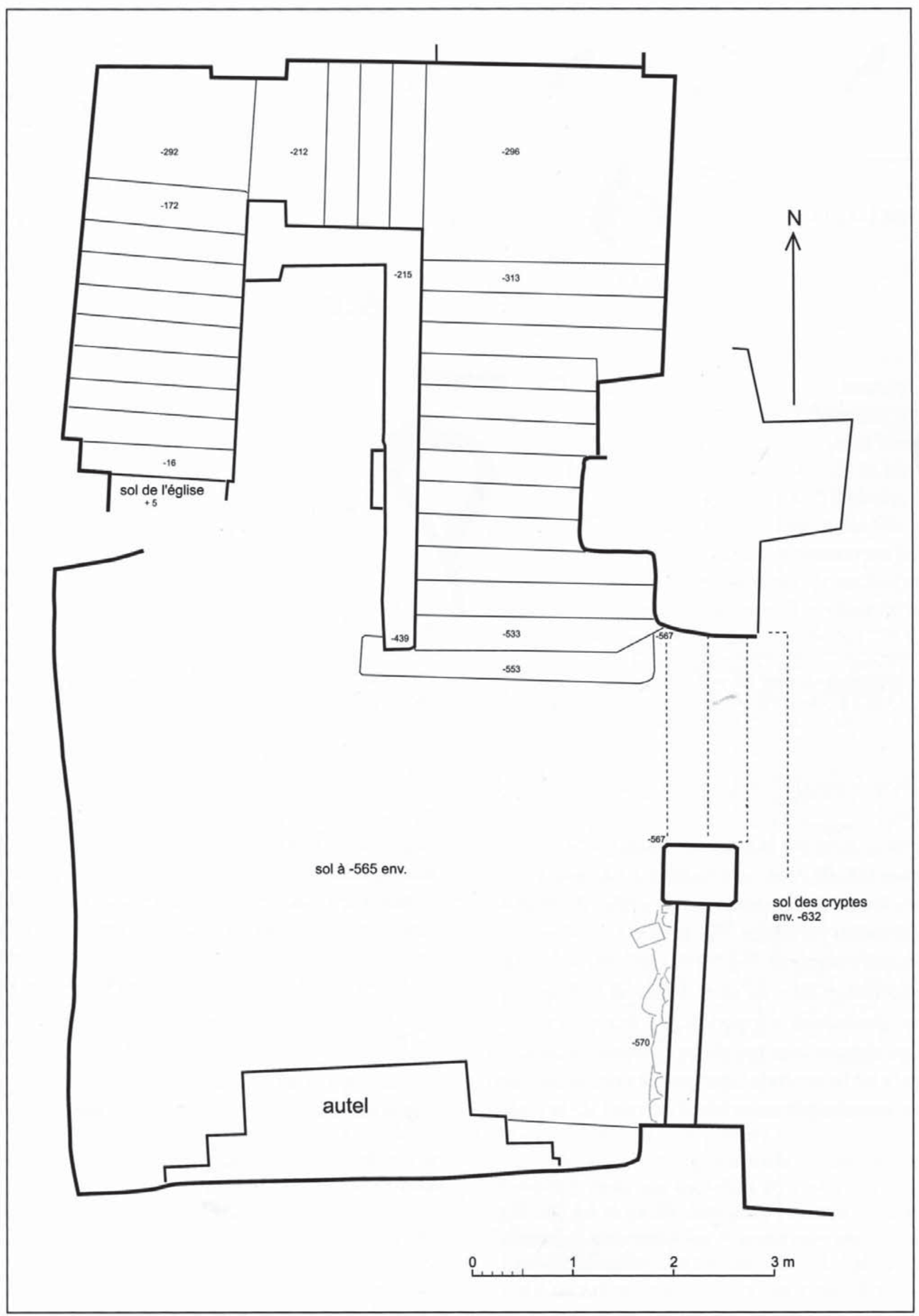

$\mathrm{Pl}$. IX - Le quatrième état de la chapelle (XVII ${ }^{e}$ siècle). 


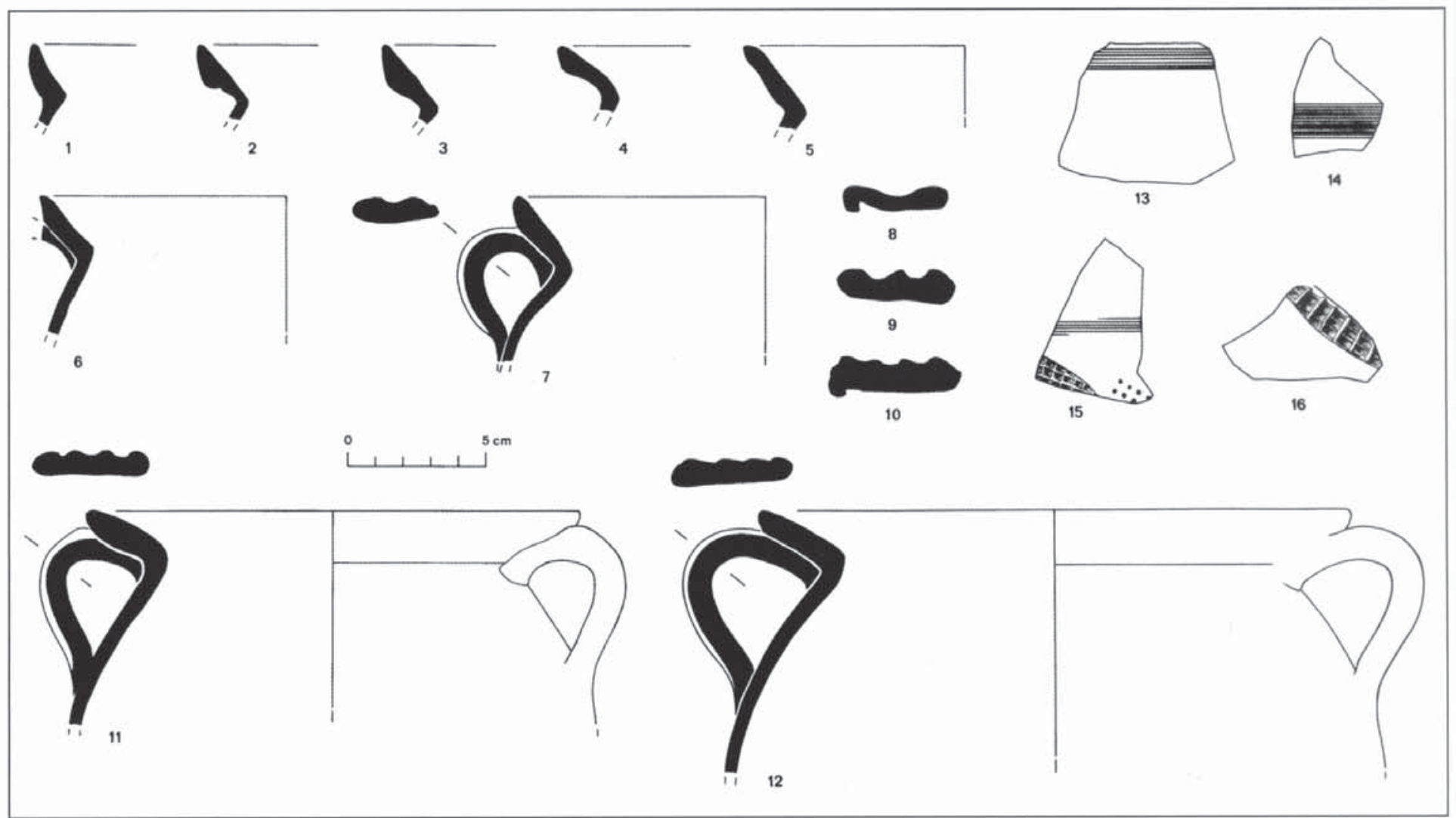

Fig. 10 - Marmites en pâte grise d'origine indéterminée (milieu XVI $-\mathrm{XVII}{ }^{\mathrm{e}}$ siècle). Profils et fragments de panses décorées au peigne (13 et 14), de cordons imprimés à la molette (16), et à la molette en oblique associée aux deux types précédents (15).

côte de Provence orientale jusqu'en Languedoc et dans la vallée du Rhône ${ }^{20}$.

Le nouvel autel, posé sur la base de l'autel médiéval a été adossé au rocher (pl. III) ; le remplissage à l'arrière contenait les mêmes types de céramiques que celles du remblai supportant le nouveau carrelage. Cet autel était intégré dans un retable baroque complexe (fig. 9) destiné primitivement à abriter le sarcophage dit " de saint Mauront ». Surmonté d'une corniche denticulée supportée par des colonnettes, il était bordé de chaque côte par deux ailerons à volutes et angelots; dans la niche centrale figuraient les statues de saint Mauront assis, encadré par saint Maurice chef de la légion thébaine et saint Elzéard de Sabran, moine à Saint-Victor au XIV' siècle On a perdu le souvenir de ces statues, conservées en place jusqu'en 1972 et remplacées par le sarcophage de Iulia Quintina ${ }^{21}$ qui retrouvait ainsi sa place. À la fin de l'ancien régime, ce sarcophage qui renfermait les restes du saint servait de corniche ou de couronnement à l'ensemble du décor baroque ${ }^{22}$. Sous la révolution, Achard le fit entrer au musée ${ }^{23}$ avec les autres sarcophages des cryptes qu'ils ont réintégré à la suite d'une décision de Gaston Defferre en $1966^{24}$.

UMR 6572, Laboratoire d'archéologie médiévale méditerranéenne-MMSH
21. M. Fixot, J.-P. Pelletier (coll. V. Gaggadis-Robin), Les sarcophages, in M.-P. Rothé, H. Treziny (dir.), Carte archéologique de la Gaule, 13/3, 2005, p. 634-644, ici p. 634.

22. Saint-Victor de Marseille, site et monument, 1, Catalogue de l'exposition par G. et D. Drocourt, Maison Diamantée-Basilique Saint-Victor, mai-septembre 1973, Marseille, 1971, Introduction par P.-A. Février,

23. W. Froehner, Musée de Marseille, Catalogue des antiquités grecques et romaines, Paris, $1897, \mathrm{XI}, \mathrm{n}^{\circ} 146$, p. 58-59.

24. Renseignements communiqués par R. Bertrand. 
BIBLIOTHÈQUE DE L'ANTIQUITÉ TARDIVE PUB LIÉE PAR L'ASSOCIATION POUR L'ANTIQUITÉ TARDIVE

\section{SAINT-VICTOR DE MARSEILLE}

\section{ÉTUDE ARCHÉOLOGIQUE ET MONUMENTALE}

Michel Fixot - Jean-Pierre Pelletier

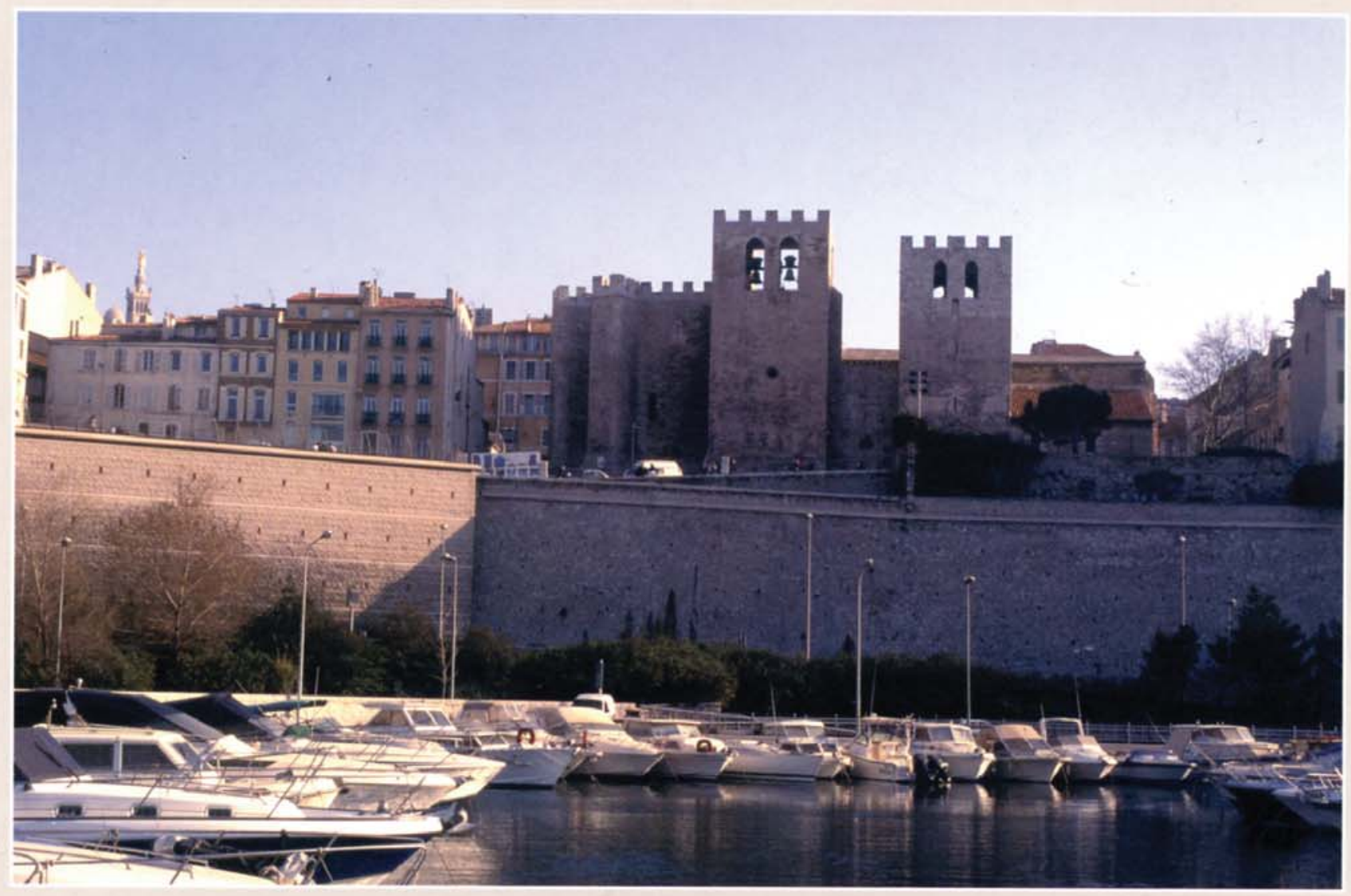



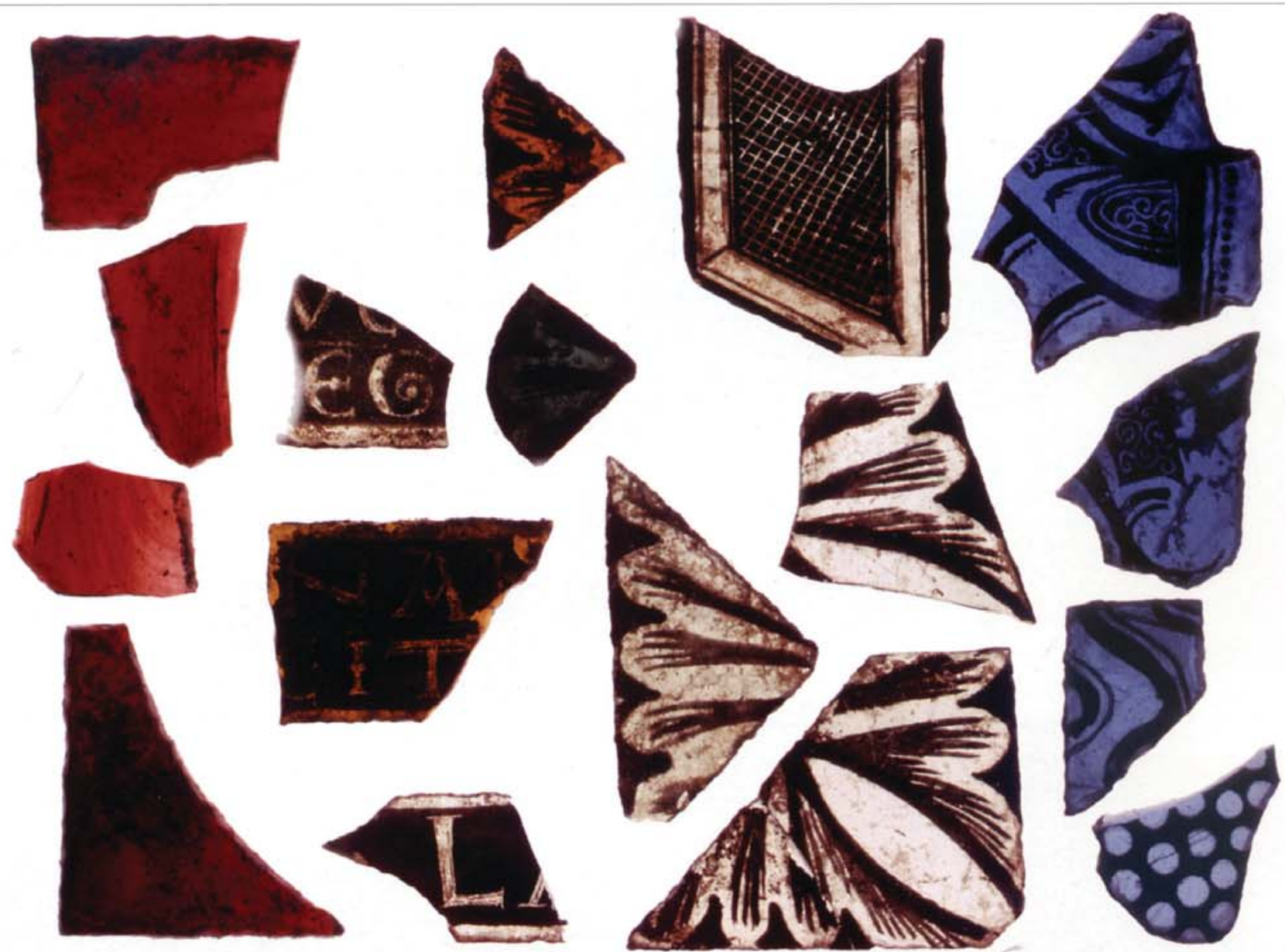

Fig. XXI - Vitraux découverts lors de la fouille du « Porche d'Isarn » (couche 3a2).

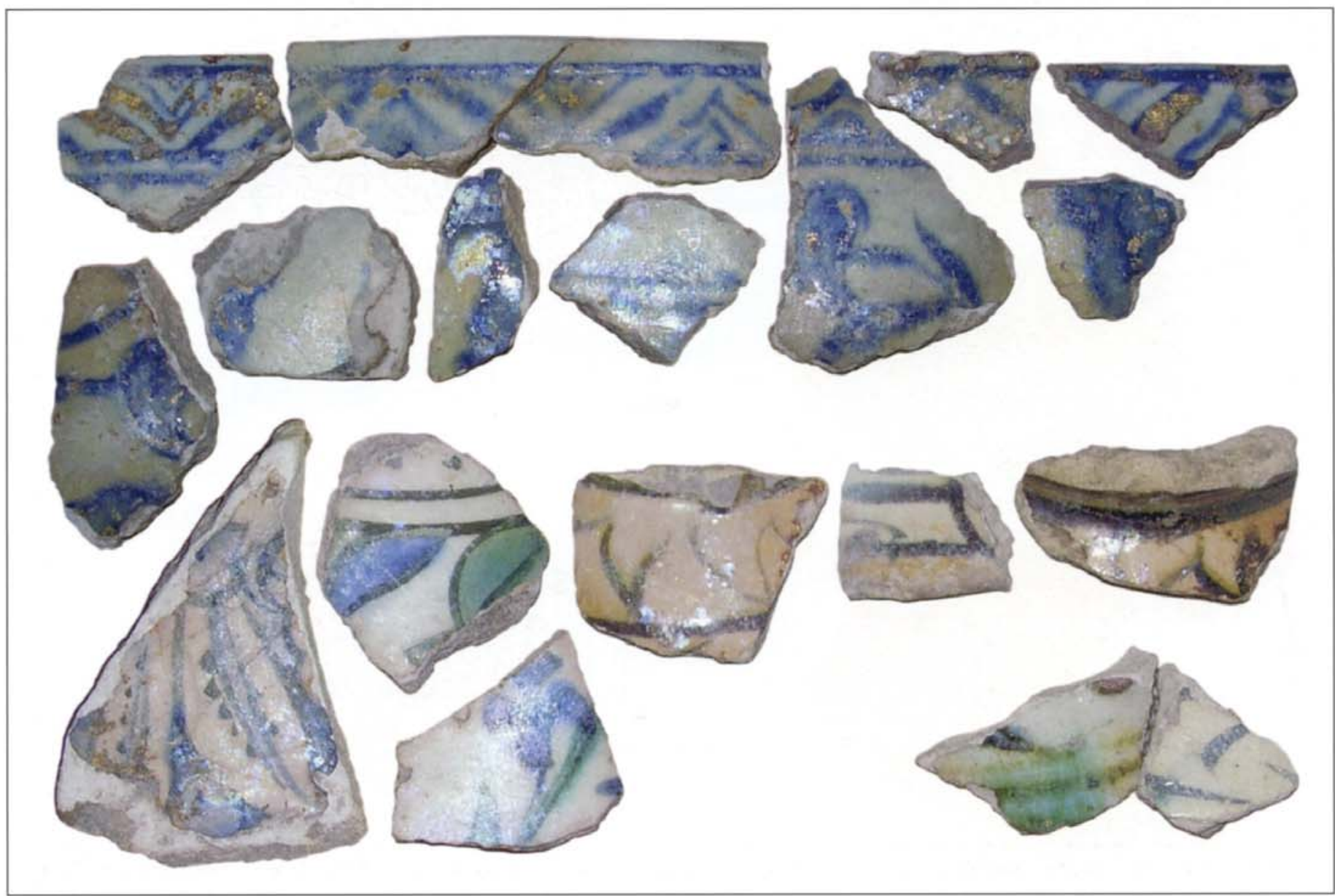

Fig. XXII - Céramiques d'Iznik (Turquie) provenant des fouilles de la chapelle Saint-Mauront : fragments d'un pot à décor bleu réhaussé d'or (en haut) et fragments de vases polychromes (fin $\mathrm{XVI}^{\mathrm{c}}$-début $\mathrm{XVII}{ }^{\mathrm{c}}$ siècle). 


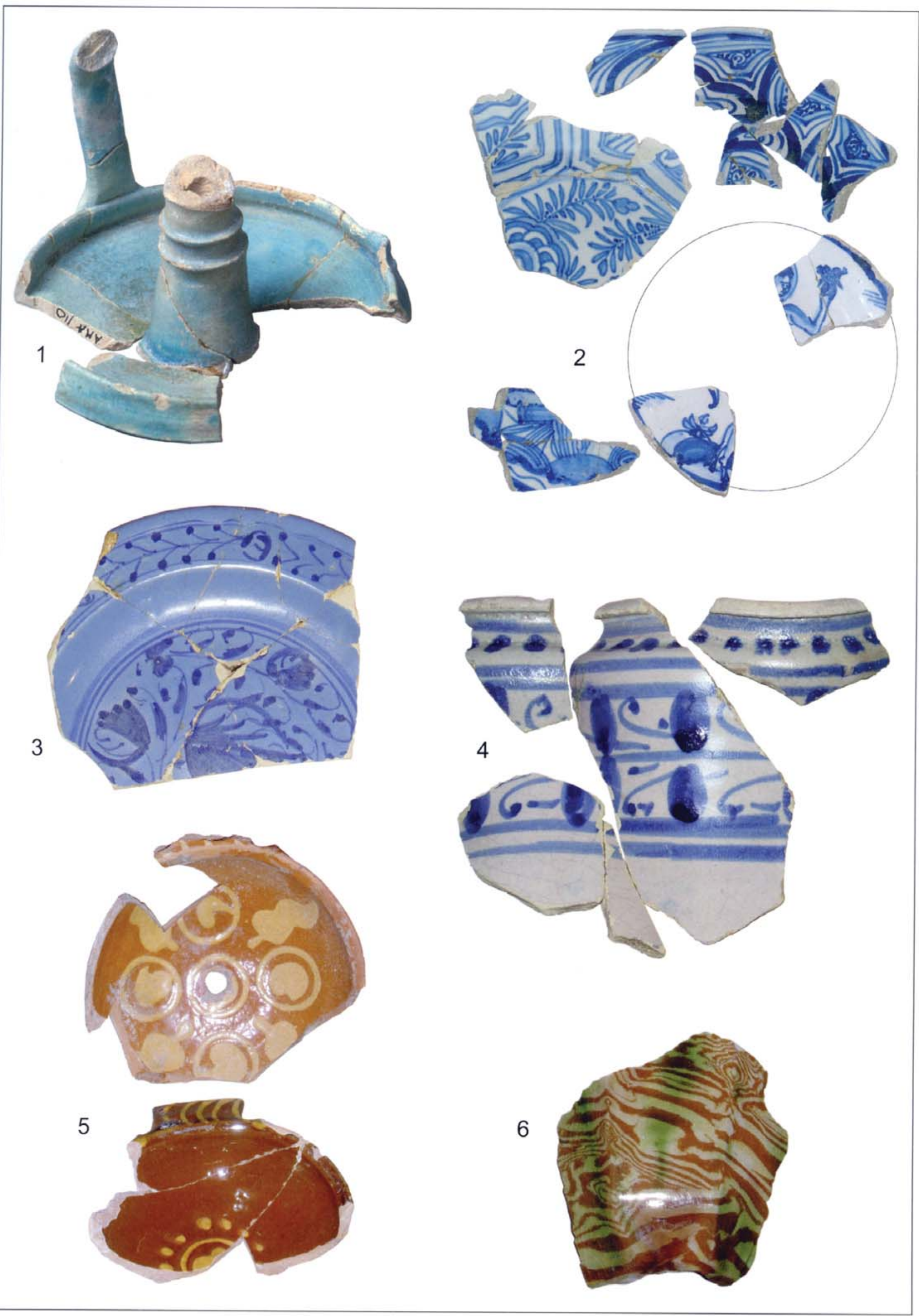

Fig. XXIII - Céramiques provenant des fouilles de la chapelle Saint-Mauront - 1. Lampe sur pied, émail turquoise (Valence, Espagne, $\mathrm{XIII}^{\circ}$ siècle) - 2. Coupes émaillées à décor de camaïeu bleu alla parcellana, dont un médaillon avec personnage (Ligurie, fin XVI ${ }^{\mathrm{e}}$-début $\mathrm{XVII}{ }^{e}$ siècle) - 3. Coupe à décor bleu sur fond émaillé bleu a berettino (Ligurie, XVII' siècle) - 4. Albarello émaillé à décor bleu (Ligurie, fin $\mathrm{XVI}^{\mathrm{e}}$-début $\mathrm{XVII}{ }^{\mathrm{e}}$ siècle) - 5. Écuelles à orielles, décor d'engobe clair sur fond brun-rouge (Provence, XVII ${ }^{\mathrm{E}}$ siècle) - 6. Panse de vase facetté en terrs mêlées (Apt ?, XVII" siècle). 